\title{
Optimal estimation and control for lossy network: stability, convergence, and performance
}

\author{
Hong Lin, Hongye Su, Senior Member, IEEE, Peng Shi, Fellow, IEEE, Zhan Shu, Member, IEEE, \\ Renquan Lu, and Zheng-Guang Wu
}

\begin{abstract}
In this paper, we study the problems of optimal estimation and control, i.e., the linear quadratic Gaussian (LQG) control, for systems with packet losses but without acknowledgment. Such acknowledgment is a signal sent by the actuator to inform the estimator of the incidence of control packet losses. For such system, which is usually called as a User Datagram Protocol (UDP)-like system, the optimal estimation is nonlinear and its calculation is time-consuming, making its corresponding optimal LQG problem complicated. We first propose two conditions: 1) the sensor has some computation abilities; and 2) the control command, exerted to the plant, is known to the sensor. For a UDP-like system satisfying these two conditions, we derive the optimal estimation. By constructing the finite and infinite product probability measure spaces for the estimation error covariances (EEC), we give the stability condition for the expected EEC, and show the existence of a measurable function to which the EEC converges in distribution, and propose some practical methods to evaluate the estimation performance. Finally, the LQG controllers are derived, and the conditions for the mean square stability of the closed-loop system are established.
\end{abstract}

Index Terms-networked control systems, optimal estimation and control, LQG, packet loss, UDP-like system, smart sensor

\section{INTRODUCTION}

\section{A. Background and Motivations}

In recent years, A great deal of attention has been devoted to networked control systems (NCSs) in which control loops are closed over network. The introduction of network does bring numerous advantages, but in the meantime, it also causes some network-induced constraints, such as limited bandwidth, quantization errors, which may result in transmission packet

Hong Lin is with the Institute of Cyber-Systems and Control, Zhejiang University, Hangzhou, Zhejiang, 310027, China; and also with the Department of Mechanical Engineering, The University of Hong Kong, Pokfulam, Hong Kong, China. (e-mail: linhongzju@163.com)

Hongye Su and Zheng-Guang Wu are with the Institute of Cyber-Systems and Control, Zhejiang University, Hangzhou, Zhejiang, 310027, China. (email: hysu@iipc.zju.edu.cn, nashwzhg@126.com)

P. Shi is with the College of Automation, Harbin Engineering University, Harbin, Heilongjiang 150001, China; and also with the College of Engineering and Science, Victoria University, Melbourne, VIC 8001, Australia. (e-mail: peng.shi@vu.edu.au)

Zhan Shu is with the Electro-Mechanical Engineering Group, Faculty of Engineering and The Environment, University of Southampton, Southampton SO17 1BJ, UK. (e-mail:hustd8@gmail.com)

Renquan Lu is with the School of Automation, Guangdong University of Technology, and Guangdong Key Laboratory of IoT Information Processing, Guangzhou 510006, China. (e-mail:rqlu@ @hdu.edu.cn)

The work is supported in part by the National Natural Science Foundation of China (61573112, U1509217, 61304072, 61673339), China National Funds for Distinguished Young Scientists (61425009), Australian Research Council (DP170102644), and the Zhejiang Provincial Natural Science Foundation of China (R1100716, LR16F030001).

Corresponding author: Hongye Su losses [1-5]. For NCSs with packet losses, there are two fundamental protocols in network communication, which are the transmission control protocol (TCP) and the user datagram protocol (UDP). The TCP features the successful data transmission by retransmitting lost data until the sending node receives a acknowledgment (ACK) signal from the receiving node. However, such retransmission mechanism in turn leads to several drawbacks, e.g., network jitter, transmission delay, additional consumption of energy and bandwidth [6]. In unreliable networks, sometimes, it would be difficult to implement the TCP by sending the ACK in time [7-9]. Thus, the TCP is commonly employed in NCSs where high security and data integrity are required and the communication is relatively reliable, such as smart grids and smart transportation. For the UDP, no ACK scheme is used and thus no retransmission of the lost data is required. Therefore, the UDP, with relatively less transmission reliability, avoids unnecessary energy consumption, causes lower latency, and allows more consistent communication, making it a preferable choice for real-time NCSs, e.g. robot control and remote teleoperations [10]. The system, in which there is no ACK signal sent from the actuator to notice the estimator the occurrence of control packet losses, is commonly called a UDP-like system (see Fig. 1-A or 1-B), and the one with ACK signals is called a TCP-like system, and the one with the ACK randomly lost is called a QuasiTCP-like system [8, 11].

It is shown in recent work $[12,13]$ that for a UDP-like system the optimal estimator is nonlinear and consisted of exponentially increasing terms, and thus its design is timeconsuming. By replacing the optimal estimator with the linear minimum-mean-square-error (LMMSE) estimator, the LQG problem, actually a sub-optimal LQG control, was studied in [14]. It is reported that for general UDP-like systems, the separation principle dose not hold, and the LQG controller is a nonlinear function of the estimated state and its solution involves solving non-convex optimization problems. Various linear sub-optimal LQG controllers were developed in [7, 15, 16]. Therefore, we may conclude that if the conventional UDP-like structure (Fig. 1-A) remains unchanged, the optimal estimation and control are not only technically involved to be obtained, but also useless in practical use due to unaffordable timeconsuming computations. It motivates us to consider whether there are some feasible or reasonable conditions or structures for UDP-like systems under which the optimal estimation and LQG control can be obtained and useful properties can be further analyzed.

Based on this motivation, we propose two mild and feasible 
conditions as follows. Condition 1: The sensor has some computation abilities, enough to run a standard Kalman filter, namely, a smart sensor; and Condition 2: The control command actually exerted to the plant (i.e., $u_{k}^{a}$ defined later) is available for the sensor. These two conditions are not so stringent that can be satisfied in some scenarios. For Condition 1, smart sensors have a wide rage of applications in many fields [17-19]. Practical techniques with complex computations_-including speech/handwriting recognition and discrete Fourier transform-have been implemented in smart sensors. In particular, the smart sensors being able to perform Kalman filtering have been wildly used to improve state estimation [20-22]. Moreover, thanks to the micro-electromechanical technology, these smart sensors can be designed to be smaller in size and lower in cost [18]. Condition 2 may be achieved in some scenarios: 1) The sensor and actuator are implemented in one device, such as the smart sensor node used in [17], in which the actuator is able to communicate and even control the sensor; 2) The actuator possesses communication ability to transfer data, such as the wireless smart actuator which can share information with the sensor [19].

In the following, we call the UDP-like system satisfying these two conditions a smart-sensor-based UDP (SS-UDP) system. Under Conditions 1 and 2 the sensor is able to obtain the information of control packet losses, that is, the ACKs. However, the way these ACKs are processed in a SS-UDP system is different from that in a Quasi-TCP-like system. In a Quasi-TCP-like system, the raw ACKs sent to the estimator over an unreliable channel is randomly lost, and thus it is required to estimate the value of $\mathrm{ACK}$, making the optimal estimator [23] and the LQG controller [8] nonlinear. In a SS-UDP system, the ACK is fused into a local estimate at the sensor side. Although the local estimate is also randomly lost over the sensor-estimator channel, the resulting optimal estimator is linear, as shown later in Sec. III-A.

It can be seen from $[1,24]$ that among the topics on optimal estimation and control for the system with packet loss, there are some closely related fundamental issues: the stability of the estimator [25, 26], the distribution and convergence of the estimation error covariance [27, 28], the estimation performance evaluation [29, 30], and the stability of the closed-loop system $[14,31]$. These four issues have been fully investigated for TCP-like systems, but they are seldom studied for SS-UDP systems. Therefore, in this paper, we are concerned with the optimal estimation and control problems for SS-UDP systems, especially the aforementioned four fundamental issues.

\section{B. Related work and contributions}

To our best knowledge, there is few work on optimal estimation and control for the proposed SS-UDP system. Recent advancements on UDP-like systems have been summarized above. In the sequel, we briefly review the state of art of these four fundamental issues for TCP-like systems.

For a conventional TCP-like system, that is, the system without a smart sensor, it is well known that its optimal estimator is the time-varying Kalman filter. Its stability was studied in the pioneering work [25] where it is pointed out that there exists a critical value which determines the boundedness of the expected estimation error covariance (EEC), i.e., $\mathbb{E}\left[\mathbf{P}_{k}\right]$. Following [25], various aspects have been further researched, including the bound for the critical value [32], the distribution for EEC [27, 28], the Markov packet losses case [26, 3335]. In [29, 30], the authors pointed out that $\mathbb{P}\left(\left\{\mathbf{P}_{k} \leq M\right\}\right)$ is a better evaluation for the estimation performance than the quantity $\mathbb{E}\left[\mathbf{P}_{k}\right]$, and then obtained the lower and upper bounds for $\mathbb{P}\left(\left\{\mathbf{P}_{k} \leq M\right\}\right)$. The LQG problem for TCP-like systems has been comprehensively investigated in [14, 31, 36, 37]. However, these results fail to apply to UDP-like or SS-UDP systems, as the structure of the optimal estimator for TCPlike systems is different from that for UDP-like or SS-UDP systems. For TCP-like systems with smart sensor, the EEC is convergent under some condition [38]. In [39], the authors proposed a LQG controller by using a smart sensor to design an encoder/decoder to compensate observation packet losses.

The introduction of smart sensors facilitates the design of the optimal estimator, however the estimation-related issues are still challenging. The resulting EEC $\mathbf{P}_{k}$ is a random quantity. To better formulate its distribution, convergent, and the limit involves an infinite product probability space. The constructions of the $\sigma$-field and the probability measure on it are complicated. Moreover, the $\mathbf{P}_{k}$ in the SS-UDP system contains $u_{k}$, and thus its properties relies on $u_{k}$, which is different from the cases in which there is no control input $[20,29,30]$. Therefore, the existing results on TCP-like systems cannot directly apply to SS-UDP systems.

In this paper, we study the optimal estimation and LQG control issues for SS-UDP systems. The main contributions are summarized as follows:

1) We construct finite and infinite product probability spaces for the estimation error covariance $\mathbf{P}_{k}$, which is the core in solving these aforementioned estimation issues.

2) We show the condition for the convergence of $\mathbf{P}_{k}$ in distribution. Moreover, we explicitly describe the limit, a measurable function on an infinite product probability space. We establish the stability condition for $\mathbb{E}\left[\mathbf{P}_{k}\right]$, and propose some practical methods to evaluate the estimation performance. For TCP-like systems these estimation issues are respectively addressed by different approaches in $[25,27,28,30]$. For SS-UDP systems we solve them in an unified way by the probability space based method.

3) For SS-UDP systems, we obtain the finite horizon LQG controller, establish the condition for the existence of the infinite horizon LQG controller, and then prove that the corresponding closed-loop system is mean square stable. It is worth noting that the optimal LQG controller for UDPlike systems has not yet been obtained; and there is no solution to the infinite horizon LQG problem for TCP-like systems as the Kalman gain is not convergent.

The rest of the paper is organized as follows: The system setup and problems are formulated in Section II. In Section III, finite and infinite product probability spaces are constructed, and then the stability, convergence, and performance evaluation of the optimal estimator are studied. The LQG problem is solved in Section IV. Conditions 1 and 2 are further discussed 
in Section V. Numerical examples are presented in Section VI to illustrate our main results. The conclusions are given in Section VII. The proofs of all lemmas and propositions are given in Appendix.

Notations:

- $\mathbb{P}(\cdot)$ denotes the probability measure.

- $\mathbb{E}[\cdot]$ denotes the probability expectation.

- $D^{c}$ denotes the complement of a set $D$.

- $(\cdot)^{\prime}$ denotes the transpose of a matrix or vector.

- Let $M$ be a matrix. $[\cdot]_{M}^{2}$ denotes $(\cdot)^{\prime} M(\cdot)$.

- $\lambda_{M}$ and $\underline{\lambda}_{M}$ stand for the maximum and the minimum singular values of $M$, respectively.

- $\|\cdot\|$ denotes the norm. Specifically, for a vector $x,\|x\| \triangleq$ $\sqrt{x^{\prime} x}$; for a matrix $M,\|M\| \triangleq \lambda_{M}$.

- $\biguplus$ stands for the union of sets and the symbol + is used to emphasize that these sets are mutually disjoint.

- $\mathbb{R}, \mathbb{N}$, and $\mathbb{Z}_{+}$denote the set of real numbers, natural numbers, and nonnegative integers, respectively.

- $\overline{\mathbb{Z}}_{+}$is defined as the set of the extend nonnegative integers, that is, $\overline{\mathbb{Z}}_{+} \triangleq \mathbb{Z}_{+} \bigcup\{\infty\}$.

- $\psi_{1} \circ \psi_{2}$ : Let $\psi_{1}$ and $\psi_{2}$ be two functions. $\psi_{1} \circ \psi_{2}(X)$ is defined as $\psi_{1}\left(\psi_{2}(X)\right)$.

- In a binary sequence, e.g., $(0,1, \times, \cdots)$, the symbol $\times$ means either 0 or 1 .

\section{SYSTEM SETUP AND PROBLEM FORMULATION}

We propose one of the possible implementations of the SSUDP system. That is the one illustrated in Fig. 1-B, in which the actuator and the smart sensor are located together in one device, like the smart sensor node used in [17], so that the actuator can provide the information $u_{k}^{a}$ to the smart sensor.

\section{A. System setup}

Consider the following discrete-time linear SS-UDP system with the framework illustrated in Fig. 1-B:

- Plant:

$$
x_{k+1}=A x_{k}+B u_{k}^{a}+\omega_{k}
$$

where $A \in \mathbb{R}^{n \times n}$ and $B \in \mathbb{R}^{n \times q}$ are constant matrices, $x_{k} \in \mathbb{R}^{n}$ is the system state, $u_{k}^{a} \in \mathbb{R}^{q}$ is the control input actually exerted to the plant by the actuator, and $\omega_{k}$ is a zero mean Gaussian noise with covariance $Q \geq 0$. In this paper, we consider the unstable system, i.e., $\lambda_{A}>1$.

- Actuator:

$$
u_{k}^{a}=\nu_{k} u_{k}
$$

where $u_{k} \in \mathbb{R}^{q}$ is the control command sent from the controller over the controller-actuator (C/A) channel. $\left\{\nu_{k}\right\}$, a sequence of i.i.d. Bernoulli random variables with $\mathbb{P}\left(\nu_{k}=1\right)=\nu$, models the control packet loss. That is, $\nu_{k}=1$ means that the control command $u_{k}$ has been successfully transmitted to the actuator, otherwise $\nu_{k}=0$.

- Sensor:

$$
y_{k}=C x_{k}+v_{k}
$$

where $C \in \mathbb{R}^{p \times n}$ is a constant matrix, $y_{k} \in \mathbb{R}^{p}$ is the observation, and $v_{k}$ is a zero mean Gaussian noises

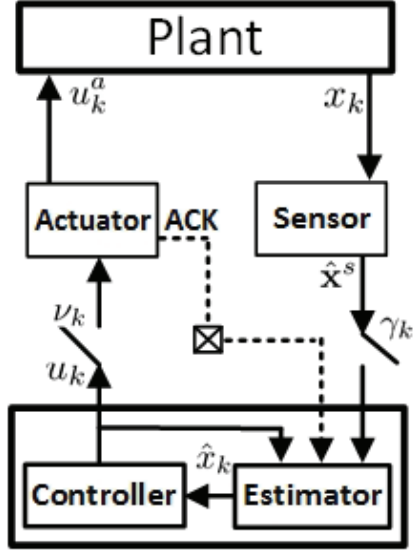

(A) The conventional UDP-like system

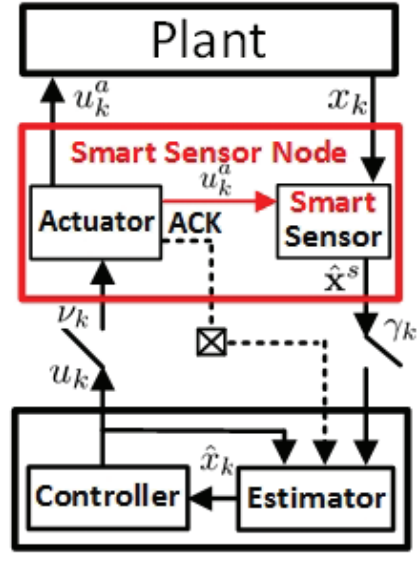

(B) The SS-UDP system
Fig. 1. The conventional UDP-like system and the SS-UDP system. The dash line with $\otimes$ indicates that there is no communication channel from the actuator to the estimator for sending acknowledgments.

with covariance $R>0$. As assumed in Condition 1 , the smart sensor is able to run a Kalman filter to obtain a local optimal state estimate $\hat{\mathbf{x}}_{k}^{s}=\mathbb{E}\left[x_{k} \mid Y_{k}\right]$, where $Y_{k}=\left\{y_{k}, \ldots, y_{1}\right\}$ (The detailed derivations are given in Section III). Then it sends $\hat{\mathbf{x}}_{k}^{s}$ instead of the raw observation $y_{k}$ to the estimator over the sensor-estimator (S/E) channel. $\left\{\gamma_{k}\right\}$, a sequence of i.i.d. Bernoulli random variables with $\mathbb{P}\left(\gamma_{k}=1\right)=\gamma$, describes the observation packet loss. Namely, $\gamma_{k}=1$ means that the local estimate $\hat{\mathbf{x}}_{k}^{s}$ has been successfully transmitted to the estimator; otherwise $\gamma_{k}=0$.

- Estimator and controller unit: Denote the information set received at the estimator side by $\mathcal{I}_{k}=\left\{\gamma_{k} \hat{\mathbf{x}}_{k}^{s}, \ldots, \gamma_{1} \hat{\mathbf{x}}_{1}^{s}\right\}$. When $\gamma_{k}=0, \gamma_{k} \hat{x}^{s}$ stands for an empty set. The task of the estimator/controller unit is to determine the optimal state estimation $\hat{\mathbf{x}}_{k}$ based on $\mathcal{I}_{k}$, and then provide the optimal control input $u_{k}$. The implementation of this unit is presented in Lemma 1 and Theorem 4, respectively.

For the system described in (1)-(3), some assumptions are given in the following.

Assumption 1. The initial state $x_{0}$ is a Gaussian random variable with mean $\bar{x}_{0}$ and covariance $P_{0} . x_{0}, \omega_{k}, v_{k}, \nu_{k}$, and $\gamma_{k}$ are mutually independent. The pair $\left(A, Q^{1 / 2}\right)$ is controllable, and the pair $(A, C)$ is observable.

\section{B. Problems formulation}

All the problems listed below are assumed to be proposed for SS-UDP systems. The aforementioned four fundamental issues are formulated in the following five problems.

\section{1) Estimation problems:}

Definition 1. An estimation of $x_{k}$, denoted by $\hat{\mathbf{x}}_{k}$, is said to be optimal in the minimum mean square error (MMSE) sense, if $\hat{\mathbf{x}}_{k}$ minimizes $\mathbb{E}\left[\left\|x_{k}-\hat{\mathbf{x}}_{k} \mid \mathcal{I}_{k}\right\|^{2}\right]$. 
Denote by $\mathbf{P}_{k}$ the estimation error covariance of $\hat{\mathbf{x}}_{k}$. It is shown later that $\mathbf{P}_{k}$ is a random variable. Although there are many convergence criteria for a random variable, a suitable one to describe $\mathbf{P}_{k}$ is the convergence in distribution. Since the probability space of $\mathbf{P}_{k}$ changes with $k$, most of the convergence criteria are not fit for this case.

Definition 2 (Convergence in distribution [40]). Let $X_{n}$ and $X$ be random variables with distributions $\mathbb{P}_{n}$ and $\mathbb{P}$, respectively. $X_{n}$ is said to converge in distribution to $X$, denoted by $X_{n} \stackrel{d}{\rightarrow}$ $X$, if $\lim _{n \rightarrow \infty} \mathbb{P}_{n}\left(\left\{X_{n}<x\right\}\right)=\mathbb{P}(\{X<x\})$ for every $x$ such that $\mathbb{P}(\{X=x\})=0$.

Problem 1. Determine the condition under which $\mathbf{P}_{k}$ converges in distribution, and find out the limit.

As $\mathbf{P}_{k}$ is a random quantity, the stability of the estimator is usually examined by the boundedness of $\mathbb{E}\left[\mathbf{P}_{k}\right]$, as in [22, $25,26]$.

Definition 3 (Stability). The estimation error covariance $\mathbf{P}_{k}$ is said to be stable in the mean sense, i.e., $\mathbb{E}\left[\mathbf{P}_{k}\right]$ is stable, if $\mathbb{E}\left[\mathbf{P}_{k}\right]$ is bounded, that is, $\sup _{k} \mathbb{E}\left[\mathbf{P}_{k}\right]<+\infty$.

Problem 2. Determine the stability condition for $\mathbb{E}\left[\mathbf{P}_{k}\right]$.

As pointed out in $[29,30], \mathbb{P}_{k}\left(\left\{\mathbf{P}_{k} \leq \alpha I\right\}\right)$ is a better evaluation of the estimation performance than $\mathbb{E}\left[\mathbf{P}_{k}\right]$, as it gives a complete characterization of the estimation performance.

Problem 3. Analyze the estimation performance in term of $\mathbb{P}_{k}\left(\left\{\mathbf{P}_{k} \leq \alpha I\right\}\right)$.

2) $L Q G$ problems: Given an integer $N$, and let $W, \Lambda, W_{N}$, $\left\{W_{k}\right\}$ and $\left\{\Lambda_{k}\right\}$ for $1 \leq k \leq N-1$ be positive definite matrices. Define the finite horizon cost function:

$$
\begin{aligned}
J_{N}\left(\pi_{N-1}, \bar{x}_{0}, P_{0}\right)= & \mathbb{E}\left[x_{N}^{\prime} W_{N} x_{N}+\sum_{k=0}^{N-1} x_{k}^{\prime} W_{k} x_{k}\right. \\
& \left.+\nu_{k} u_{k}^{\prime} \Lambda_{k} u_{k} \mid \pi_{N-1}, \bar{x}_{0}, P_{0}\right]
\end{aligned}
$$

and the infinite horizon cost function:

$$
\begin{aligned}
J_{\infty}\left(\pi_{\infty}, \bar{x}_{0}, P_{0}\right) & =\lim _{N \rightarrow \infty} \frac{1}{N} \mathbb{E}\left[\sum_{k=0}^{N-1} x_{k}^{\prime} W x_{k}\right. \\
& \left.+\nu_{k} u_{k}^{\prime} \Lambda u_{k} \mid \pi_{N-1}, \bar{x}_{0}, P_{0}\right]
\end{aligned}
$$

where $\pi_{N-1}=\left\{u_{0}, \ldots, u_{N-1}\right\}$ and $\pi_{\infty}=\left\{u_{0}, \ldots, u_{k}, \ldots\right\}$ stand for sequences of control inputs. Each $u_{k}$ is a function of $\mathcal{I}_{k}$, i.e., $u_{k}=f_{k}\left(\mathcal{I}_{k}\right)$. For the optimal control, some assumptions are given as follows:

Assumption 2. The pair $(A, B)$ is controllable, and the pair $\left(A, W^{1 / 2}\right)$ is observable.

Problem 4. Determine the optimal control sequence, denoted by $\pi_{N-1}^{*}\left(\right.$ or $\left.\pi_{\infty}^{*}\right)$, minimizing the cost function $J_{N}\left(\right.$ or $\left.J_{\infty}\right)$,

$$
\begin{aligned}
& J_{N}^{*} \triangleq J_{N}\left(\pi_{N-1}^{*}, \bar{x}_{0}, P_{0}\right)=\min _{\pi_{N-1}} J_{N}\left(\pi_{N-1}, \bar{x}_{0}, P_{0}\right) \\
& J_{\infty}^{*} \triangleq J_{\infty}\left(\pi_{\infty}^{*}, \bar{x}_{0}, P_{0}\right)=\min _{\pi_{\infty}} J_{N}\left(\pi_{\infty}, \bar{x}_{0}, P_{0}\right) .
\end{aligned}
$$

The closed-loop system, whenever mentioned in the following, refers to the SS-UDP system with the feedback control
$u_{k}=L_{\infty} \hat{\mathbf{x}}_{k}$, where $L_{\infty}$ is the infinite horizon LQG control gain. The closed-loop system is consisted of three dynamic subsystems, whose states are the plant $x_{k}$, the smart sensor $\hat{\mathbf{x}}_{k}^{s}$, and the estimator $\hat{\mathbf{x}}_{k}$, respectively. It has been pointed out in [31] that even when the infinite LQG control exists, the controller does not necessarily stabilize the closed-loop system. Hence, we consider the following problem.

Definition 4 (Mean square stability (MMS)). The closed-loop system is said to be mean square (m.s.) stable, if $\mathbb{E}\left[\left\|x_{k}\right\|^{2}\right]<$ $+\infty, \mathbb{E}\left[\left\|\hat{\mathbf{x}}_{k}\right\|^{2}\right]<+\infty$, and $\mathbb{E}\left[\left\|\hat{\mathbf{x}}_{k}^{s}\right\|^{2}\right]<+\infty$ for all $k \in \mathbb{N}$.

Problem 5. Determine the condition under which the closedloop system is m.s. stable.

Our objectives are to solve these five problems above. The following table provides a reference for their answers. The column of main topics explains in which problem the terms "stability, convergence, and performance" in the title of this paper are involved.

\begin{tabular}{ccc} 
Problems & Answers & Main topics \\
\hline 1 & Theorem 1 & Convergence \\
2 & Theorem 2 & Stability \\
3 & Theorem 3 & Estimation Performance \\
4 & Theorems 4 and 5(i) & LQG control \\
5 & Theorem 5(ii) & Stability \\
\hline
\end{tabular}

\section{OPTIMAL ESTIMATOR AND ITS PROPERTIES}

\section{A. Smart sensor based optimal estimator}

As assumed in Condition 1, the smart sensor is able to run standard Kalman filter to obtain the local optimal estimate $\hat{\mathbf{x}}_{k}^{s}$ and its corresponding estimation error covariance $S_{k}$ as follows:

$$
\begin{aligned}
\overline{\mathbf{x}}_{k+1}^{s} & =A \hat{\mathbf{x}}_{k}^{s}+\nu_{k} B u_{k}=A \hat{\mathbf{x}}_{k}^{s}+B u_{k}^{a} \\
\bar{S}_{k+1} & =A S_{k} A^{\prime}+Q \\
K_{k+1} & =\bar{S}_{k+1} C^{\prime}\left(C \bar{S}_{k+1} C^{\prime}+R\right)^{-1} \\
\hat{\mathbf{x}}_{k+1}^{s} & =\overline{\mathbf{x}}_{k+1}^{s}+K_{k+1}\left(y_{k+1}-C \overline{\mathbf{x}}_{k+1}^{s}\right) \\
S_{k+1} & =\left(I-K_{k+1} C\right) \bar{S}_{k+1},
\end{aligned}
$$

with $\hat{\mathbf{x}}_{0}^{s}=\bar{x}_{0}$ and $S_{0}=P_{0}$.

Lemma 1. For the SS-UDP system, the optimal estimator is the following.

$$
\begin{aligned}
& \hat{\mathbf{x}}_{k}=\gamma_{k} \hat{\mathbf{x}}_{k}^{s}+\left(1-\gamma_{k}\right)\left(A \hat{\mathbf{x}}_{k-1}+\nu B u_{k-1}\right) \\
& \mathbf{P}_{k}=\gamma_{k} S_{k}+\left(1-\gamma_{k}\right)\left(A \mathbf{P}_{k-1} A^{\prime}+Q+U_{k-1}\right),
\end{aligned}
$$

with $\hat{\mathbf{x}}_{0}=\bar{x}_{0}$ and $\mathbf{P}_{0}=P_{0}$, where $U_{k-1} \triangleq \bar{\nu} \nu B u_{k-1} u_{k-1}^{\prime} B^{\prime}$ and $\bar{\nu}=1-\nu$.

\section{B. Construction of probability spaces}

Problems 1, 2, and 3 in Section II are closely related to $\mathbf{P}_{k}$. From (8), it is clear that $\mathbf{P}_{k}$ is a random variable. The probability space of $\mathbf{P}_{k}$ and its limit play an important role in solving these problems. Therefore, in this section, we first construct the finite product probability space $\left(\Omega_{k}, \mathscr{F}_{k}, \mathbb{P}_{k}\right)$ of $\mathbf{P}_{k}$. Then we construct an infinite product probability space $\left(\Omega_{\infty}, \mathscr{F}_{\infty}, \mathbb{P}_{\infty}\right)$-which characterizes the behavior of the limit 
of $\mathbb{P}_{k}$-and a measurable function (i.e., a random variable) on it. Based on them, these problems are solved in Section III-C, $-\mathrm{D}$, and -E, respectively.

We start with some definitions relevant to the probability space.

Definition 5 ([41]). Some definitions relevant to field are given as follows.

- (Field and $\sigma$-field) Let $\mathscr{F}$ be a collection of subsets of a set $\Omega$. Then $\mathscr{F}$ is called a field if (i) $\Omega \in \mathscr{F}$; (ii) If $A \in \mathscr{F}$, then $A^{c} \in \mathscr{F}$; (iii) If $A_{1}, \ldots, A_{n} \in \mathscr{F}$, $\bigcup_{i=1}^{n} A_{i} \in \mathscr{F} . \mathscr{F}$ is called a $\sigma$-field, if (iii) is replaced by "If $A_{1}, A_{2}, \ldots \in \mathscr{F}, \bigcup_{i=1}^{\infty} A_{i} \in \mathscr{F}$."

- ( $\sigma$-field generated by sets) Let $\mathscr{L}$ be a collection of subsets of $\Omega$, a $\sigma$-field $\mathscr{F}_{0}$ is called the $\sigma$-field generated by $\mathscr{L}$, denoted by $\sigma(\mathscr{L})$, if $\mathscr{F}_{0}$ is included in any $\sigma$-field containing $\mathscr{L}$.

Definition 6 ([41]). Some definitions relevant to measure are given as follows.

- (Finitely and countably additive) Let $\mathscr{F}$ be a $\sigma$-field of a set $\Omega$. A set function $\mu: \mathscr{F} \rightarrow \mathbb{R}$ is called countably additive on $\mathscr{F}$, if whenever $A_{1}, A_{2}, \ldots$ form a finite or countable collection of disjoint sets in $\mathscr{F}$ whose union also belong to $\mathscr{F}$, we have $\mu\left(\bigcup_{n} A_{n}\right)=\sum_{n} \mu\left(A_{n}\right)$. If this equity holds only for finite collections of disjoint sets in $\mathscr{F}$, then $\mu$ is called finitely additive.

- (Measure) A measure on a $\sigma$-field (or field) is a nonnegative real value countably additive set function $\mu$. If $\mu(\Omega)=1, \mu$ is called a probability measure, usually denoted by $\mathbb{P}$.

- (Probability space) A probability space is a triple $(\Omega, \mathscr{F}, \mathbb{P})$, where $\Omega$ is a set and is usually called the sample space, $\mathscr{F}$ is a $\sigma$-field on $\Omega$, and $\mathbb{P}$ is probability measure on $\mathscr{F}$.

- (Measurable function) Let $\left(\Omega_{1}, \mathscr{F}_{1}\right)$ and $\left(\Omega_{2}, \mathscr{F}_{2}\right)$ be measurable spaces. A function $h: \Omega_{1} \rightarrow \Omega_{2}$ is said to be $\mathscr{F}_{1}-\mathscr{F}_{2}$ measurable (or measurable for short) if $h^{-1}(A) \in \mathscr{F}_{1}$ for each $A \in \mathscr{F}_{2}$.

- $(\sigma$-finite) A nonnegative, finitely additive set function $\mu$ on the field $\mathscr{F}_{1}$ is called $\sigma$-finite if $\Omega$ can be written as $\bigcup_{i=1}^{\infty} A_{i}$ where $A_{i} \in \mathscr{F}_{1}$ and $\mu\left(A_{i}\right)<\infty$.

1) Probability space $\left(\Omega_{k}, \mathscr{F}_{k}, \mathbb{P}_{k}\right):\left\{\gamma_{k}\right\}$ is a sequence of i.i.d. Bernoulli random variables. According to the definition above, we denote the probability space for each $\gamma_{k}$ by $\left(\Omega_{\gamma}, \mathscr{F}_{\gamma}, \mathbb{P}_{\gamma}\right)$ where $\Omega_{\gamma}=\{0,1\}, \mathscr{F}_{\gamma}$ is the conventional $\sigma$-field for $\Omega_{\gamma}$, i.e., $\mathscr{F}_{\gamma}=\left\{\phi, \Omega_{\gamma},\{0\},\{1\}\right\} . \mathbb{P}_{\gamma}$ is the probability measure on $\mathscr{F}_{\gamma}$ with $\mathbb{P}_{\gamma}\left(\gamma_{k}=1\right)=\gamma$, where the subscript $\gamma$ is used to distinguish those $\mathbb{P}_{k}$ and $\mathbb{P}_{\infty}$ defined later. In the sequel, we construct the probability space of $\mathbf{P}_{k}$.

$\Omega_{k}$ : It can be seen from (8) that $\mathbf{P}_{k}$ depends on $\left(\gamma_{k}, \ldots, \gamma_{1}\right)$, which may take $2^{k}$ different values. The sample space $\Omega_{k}$ is the set consisting of all these $2^{k}$ points. The point in $\Omega_{k}$ is denoted by $\theta \triangleq\left(\gamma_{k}, \ldots, \gamma_{1}\right)$.

$\mathscr{F}_{k}$ : We take the $\sigma$-field $\mathscr{F}_{k}$ to consist of all subsets of $\Omega_{k}$, i.e., $\mathscr{F}_{k} \triangleq\left\{A \subseteq \Omega_{k}\right\}$.

$\mathbb{P}_{k}$ : The probability measure $\mathbb{P}_{k}$ is a set function: $\mathscr{F}_{k} \rightarrow$
$[0,1]$, which can be defined in the following way. For each single point set $\{\theta\}=\left\{\left(\gamma_{k}, \ldots, \gamma_{1}\right)\right\} \subseteq$ $\Omega_{k}$, due to the mutual independence of $\left(\gamma_{k}, \ldots, \gamma_{1}\right)$, $\mathbb{P}_{k}(\{\theta\})=\prod_{i=1}^{k} \mathbb{P}_{\gamma}\left(\gamma_{i}\right)$. Then we can assign each set $A \in \mathscr{F}_{k}$ a probability measure in such a way that $\mathbb{P}_{k}(A) \triangleq \sum_{\theta_{i} \in A} \mathbb{P}_{k}\left(\left\{\theta_{i}\right\}\right)$. It is easy to check that $\mathbb{P}_{k}$ is indeed a probability measure.

2) Random variable $\mathbf{P}_{k}$ : As a random variable, $\mathbf{P}_{k}$ is in fact a measurable function mapping $\Omega_{k}$ to $\mathbb{R}^{n \times n}$. To better describe $\mathbf{P}_{k}$, we will define some subsets of $\Omega_{k}$. On each subset, $\mathbf{P}_{k}$ takes a constant value.

Recall that $\Omega_{k}$ consists of points of the form $\theta=$ $\left(\gamma_{k}, \ldots, \gamma_{1}\right)$. We define $F_{k}^{[k]} \triangleq\{(0, \ldots, 0)\}$, and for $0 \leq$ $i \leq k-1$,

$$
F_{k}^{[i]} \triangleq\left\{\begin{array}{ccccccc}
(0 & \ldots & 0 & 1 & \times & \ldots & \times \\
1 & \ldots & i & i+1 & i+2 & \ldots & k
\end{array}\right\}
$$

where from left to right the first " 1 " occurs in the $i+1$ th position. That is, for each $\theta \in F_{k}^{[j]}$, we have

$$
\theta=\left\{\gamma_{k}=0, \ldots, \gamma_{k-j+1}=0, \gamma_{k-j}=1, \times, \ldots, \times\right\} .
$$

Take $k=3$ for example:

$F_{3}^{[0]}=\{(1,0,0),(1,0,1),(1,1,0),(1,1,1)\}, F_{3}^{[1]}=$ $\{(0,1,0),(0,1,1)\}, F_{3}^{[2]}=\{(0,0,1)\}, F_{3}^{[3]}=\{(0,0,0)\}$.

Proposition 1. For the subsets $F_{k}^{[i]}$, the following facts hold.

(i) $F_{k}^{[i]} \cap F_{k}^{[j]}=\emptyset$ for $0 \leq i \neq j \leq k$ ( $\emptyset$ means an empty set).

(ii) $\Omega_{k}=\biguplus_{i=0}^{k} F_{k}^{[i]}$.

(iii) $\mathbb{P}_{k}\left(F_{k}^{[j]}\right)=\gamma \bar{\gamma}^{j}$ for $0 \leq j \leq k-1$, and $\mathbb{P}_{k}\left(F_{k}^{[k]}\right)=\bar{\gamma}^{k}$.

Define two functions $\psi_{k}(P)$ and $\Psi_{k}^{[i]}(P)$ as follows:

$$
\begin{aligned}
\psi_{k}(P) & =A P A^{\prime}+Q+U_{k} \\
\Psi_{k}^{[i]}(P) & = \begin{cases}P, & \text { for } i=0 \\
\psi_{k-1} \circ \cdots \circ \psi_{k-i}(P), & \text { for } 1 \leq i \leq k\end{cases}
\end{aligned}
$$

For $0 \leq i \leq k$, we define

$$
P_{k}^{[i]} \triangleq \Psi_{k}^{[i]}\left(S_{k-i}\right),
$$

where $S_{k}$ is computed by (5) and (6).

Lemma 2. For $\forall k$ and $\forall\left\{U_{k}\right\}, P_{k}^{[n]} \leq P_{k}^{[n+1]}, 0 \leq n \leq k-1$.

Proposition 2. As a function, $\mathbf{P}_{k}$ can be described as follows:

$$
\mathbf{P}_{k}(\theta)=P_{k}^{[j]}, \text { for } \theta \in F_{k}^{[j]} \subseteq \Omega_{k} .
$$

3) Probability space $\left(\Omega_{\infty}, \mathscr{F}_{\infty}, \mathbb{P}_{\infty}\right)$ : As $k \rightarrow \infty, \Omega_{k}$ becomes an infinite product space $\Omega_{\infty} \triangleq \Omega_{\gamma} \times \Omega_{\gamma} \times \cdots$. To study the behavior of the limit of $\mathbf{P}_{k}$, we construct the probability space $\left(\Omega_{\infty}, \mathscr{F}_{\infty}, \mathbb{P}_{\infty}\right)$ as follows.

$\Omega_{\infty}$ : Since $\Omega_{\infty}=\Omega_{\gamma} \times \Omega_{\gamma} \times \cdots$, it consists of infinitely many points and each point $\theta \in \Omega_{\infty}$ takes the form $\theta=\left(\theta_{1}, \ldots, \theta_{i}, \ldots\right)$ with $\theta_{i}=0$ or 1 for $i \in \mathbb{N}$.

$\mathscr{F}_{\infty}$ : Clearly, $\Omega_{\infty}$ is an uncountable set. A well known result in the measure theory is that if the $\sigma$-field consists of all the subsets of an uncountable set $\Omega_{\infty}$, then there is no measure on the $\sigma$-field [40]. 
Therefore, we have to equip $\Omega_{\infty}$ with a suitable $\sigma$ field and then construct a probability measure on it. Define some countable subsets of $\Omega_{\infty}$ as follows: Let $A=\{(0,0, \ldots)\}$, and let

$$
\begin{aligned}
& A^{[i]}=\left\{\left(\begin{array}{ccccccc}
0 & \ldots & 0 & 1 & \times & \ldots
\end{array}\right)\right\} . \\
& 1 \ldots . \quad i \quad i+1 \quad i+2 \quad \ldots
\end{aligned}
$$

For the convenience of indexing the sets $A$ and $A^{[i]}$, we define $A^{[\infty]} \triangleq A$. Let $\mathscr{A}_{0}$ be a collection of sets $\left\{\emptyset, \Omega_{\infty}, A^{[0]}, A^{[1]}, \ldots, A^{[\infty]}\right\}$, and let $\mathscr{A}$ be a collection of all the finite and countable unions of subsets of $\mathscr{A}_{0}$. That is, if $B \in \mathscr{A}$, there exists a finite or countable set

$$
\mathcal{N}_{B}=\left\{n_{1}, n_{2}, \ldots\right\} \subseteq \overline{\mathbb{Z}}_{+}
$$

such that $B=\biguplus_{i \in \mathcal{N}_{B}} A^{[i]}$.

Define $\mathscr{F}_{\infty}=\sigma(\mathscr{A})$, the $\sigma$-field generated by $\mathscr{A}$.

$\mathbb{P}_{\infty}$ : Generally, it is difficult to explicitly define a measure $\mathbb{P}$ by assigning a value for every set in $\mathscr{F}_{\infty}$. Conventionally, one can define a desired measure $\mathbb{P}^{*}$ on some subsets of interest, such as $\mathscr{A}$, and then extend $\mathbb{P}^{*}$ to a probability measure $\mathbb{P}$ on $\mathscr{F}_{\infty}$. Such $\mathbb{P}$ is identical to $\mathbb{P}^{*}$ when acting on $\mathscr{A}$. Following this idea, we define a set function $\mathbb{P}^{*}: \mathscr{A} \rightarrow[0,1]$ in such a way that

$$
\mathbb{P}^{*}\left(A^{[\infty]}\right)=0, \text { and } \mathbb{P}^{*}\left(A^{[i]}\right)=\gamma \bar{\gamma}^{i} \triangleq p_{i},
$$

and

$$
\mathbb{P}^{*}(B) \triangleq \sum_{i \in \mathcal{N}_{B}} p_{i}, \text { for } B \in \mathscr{A},
$$

where $B=\biguplus_{i \in \mathcal{N}_{B}} A^{[i]}$. The existence of $\mathbb{P}$ on $\mathscr{F}_{\infty}$ is formulated later in Proposition 5.

The following proposition lists some properties of the structure of $\mathscr{A}$.

Proposition 3. The following facts hold.

(i) $A^{[i]} \cap A^{[j]}=\emptyset$ for $i \neq j \in \overline{\mathbb{Z}}_{+}$.

(ii) $\Omega_{\infty}=\biguplus_{i \in \overline{\mathbb{Z}}_{+}}^{\infty} A^{[i]}$, i.e., $\Omega_{\infty}=A \biguplus\left(\biguplus_{i=0}^{\infty} A^{[i]}\right)$.

(iii) Given a set $B \in \mathscr{A}$, the set $\mathcal{N}_{B}$ mentioned in (12) is unique. If $B_{i}$ and $B_{j}$ are disjoint, so are $\mathcal{N}_{B_{i}}$ and $\mathcal{N}_{B_{j}}$. Moreover, $B_{i} \biguplus B_{j}=\biguplus_{k \in \mathcal{N}_{B_{i}} \biguplus \mathcal{N}_{B_{j}}} A^{[k]}$.

(iv) Let $\left\{B_{i}\right\}$ be a sequence of mutually disjoint sets. Then

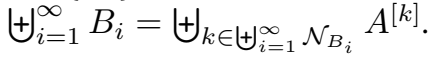

To extend $\mathbb{P}^{*}$ to a probability measure on $\mathscr{F}_{\infty}$, we require the famous measure extension theorem as follows.

Lemma 3 (Carathéodory measure extension theorem [41]). Let $\Omega^{*}$ be a nonempty set, and let $\mu^{*}$ be a measure on the field $\mathscr{F}_{0}$ of subsets of $\Omega^{*}$, and assume that $\mu^{*}$ is $\sigma$-finite on $\mathscr{F}_{0}$, then $\mu^{*}$ has an unique extension to a measure $\mu$ on $\sigma\left(\mathscr{F}_{0}\right)$ such that $\mu(A)=\mu^{*}(A)$ for $A \in \mathscr{F}_{0}$.

The following proposition shows that $\mathscr{A}$ and $\mathbb{P}^{*}$ meet the requirements in the preceding measure extension theorem.

Proposition 4. The following facts hold. (i) $\mathscr{A}$ is a field; (ii) $\mathbb{P}^{*}$ is a measure on the field $\mathscr{A}$; and (iii) $\mathbb{P}^{*}$ is $\sigma$-finite on $\mathscr{A}$.
Based on Propositions 3, 4 and the measure extension theorem, the existence of the probability measure $\mathbb{P}$ on $\mathscr{F}_{\infty}$ is shown as follows.

Proposition 5. There exists a probability measure $\mathbb{P}_{\infty}$ on $\mathscr{F}_{\infty}$ such that $\mathbb{P}_{\infty}(A)=\mathbb{P}^{*}(A)$ for $\forall A \in \mathscr{A}_{0}$, that is, $\mathbb{P}_{\infty}(\emptyset)=0$, $\mathbb{P}_{\infty}\left(\Omega_{\infty}\right)=1$, and $\mathbb{P}_{\infty}\left(A^{[i]}\right)=\mathbb{P}^{*}\left(A^{[i]}\right)$ for $\forall i \in \overline{\mathbb{Z}}_{+}$.

4) Construction of the random variable $\mathbf{P}$ on $\Omega_{\infty}$ : In the following, we construct a random variable $\mathbf{P}$ on $\left(\Omega_{\infty}, \mathscr{F}_{\infty}, \mathbb{P}_{\infty}\right)$, which will be the limit of $\mathbf{P}_{k}$. For a probability space, a random variable is in fact a measurable function. Hence, to construct $\mathbf{P}$, we only need to designate its domain and the values it takes. Define two functions

$$
\begin{aligned}
\psi(P) & =A P A^{\prime}+Q+U \\
\Psi^{[m]}(P) & = \begin{cases}P, & \text { for } m=0 \\
\underbrace{\psi \circ \cdots \circ \psi}_{m \text { times }}(P), & \text { for } m \geq 1 .\end{cases}
\end{aligned}
$$

It follows from [42, Theorem 17.53] that under Assumption 1 $\bar{S}_{k}$ and $S_{k}$ in (5) and (6) converge, and their limits, denoted by $\bar{S}$ and $S$ respectively, are positive define. Define

$$
S^{[m]} \triangleq \Psi^{[m]}(S), \text { for } m \geq 0
$$

and then define a random variable $\mathbf{P}: \Omega_{\infty} \mapsto \mathbb{R}^{n \times n}$ as

$$
\mathbf{P}(\theta)= \begin{cases}0, & \text { for } \theta \in A^{[\infty]} \\ S^{[m]}, & \text { for } \theta \in A^{[m]}, m \in \mathbb{Z}_{+} .\end{cases}
$$

From the definition above, the range of $\mathbf{P}$ is countable. For any subset $M \subseteq \mathbb{R}^{n \times n}$, the inverse image $\mathbf{P}^{-1}(M)$ consists of finite or countable subsets $A^{[i]}$, i.e., the union of these subsets $A^{[i]}\left(\mathbf{P}^{-1}(M)=\biguplus A^{[i]}\right)$. From the construction of $\mathscr{A}$, it is clear that $\biguplus A^{[i]} \in \mathscr{A}$, which indicates that $\mathbf{P}$ is a measurable function, that is, a random variable. To formulate the distribution of $\mathbf{P}$, we give a lemma as follows.

Denote the maximum singular values of $S^{[m]}$ and $P_{k}^{[m]}$ by $\lambda_{S}^{[m]}$ and $\lambda_{P_{k}}^{[m]}$, respectively.

\section{Lemma 4. The following facts hold.}

(i) $\left\{S^{[n]}\right\}$ for $n \in \mathbb{Z}_{+}$is a strictly increasing sequence.

(ii) $\lambda_{S}^{[n]}$ is an increasing sequence, and $\lim _{n \rightarrow \infty} \lambda_{S}^{[n]}=\infty$.

(iii) For given $\alpha \geq \lambda_{S}$ and $U \geq 0$, there exists an unique integer $n_{\alpha} \in \mathbb{N}$ such that $\bar{\lambda}_{S}^{\left[n_{\alpha}\right]} \leq \alpha<\lambda_{S}^{\left[n_{\alpha}+1\right]}$. The following function is well-defined

$$
n_{\alpha}=n(\alpha, U) \text {. }
$$

For brevity, denote the subset $\left\{\theta \in \Omega_{k} \mid \mathbf{P}_{k}(\theta) \leq \alpha I\right\}$ by $\left\{\mathbf{P}_{k} \leq \alpha I\right\}$ and $\left\{\theta \in \Omega_{\infty} \mid \mathbf{P}(\theta) \leq \alpha I\right\}$ by $\{\mathbf{P} \leq \alpha I\}$.

Proposition 6. The distribution of $\mathbf{P}$ is the following.

(i) $\mathbb{P}_{\infty}\left(\left\{\mathbf{P}=S^{[m]}\right\}\right)=\gamma \bar{\gamma}^{m}$ for $m \in \overline{\mathbb{Z}}_{+\cdot}\left(\gamma \bar{\gamma}^{\infty} \triangleq 0\right)$

(ii) $\mathbb{P}_{\infty}(\{\mathbf{P} \leq \alpha I\})=1-\bar{\gamma}^{n_{\alpha}+1}$, where $n_{\alpha}=n(\alpha, U)$ and $\alpha \geq \lambda_{S}$. 


\section{Convergence of $\mathbf{P}_{k}$}

Based on the probability spaces constructed above, we deal with Problem 1 in this section, and the result is formulated in the following theorem.

Theorem 1. If $U_{k}$ is convergent, then $\mathbf{P}_{k}$ converges to $\mathbf{P}$ in distribution, i.e., $\mathbf{P}_{k} \stackrel{d}{\rightarrow} \mathbf{P}$, where $\mathbf{P}$ is constructed by (15)(18).

To prove Theorem 1, some lemmas are given as follows.

Lemma 5. Let $X, Y$, and $S$ be matrices where $S>0$, and let $\alpha>0$ and $\varepsilon>0$ be real numbers. The following facts hold.

(i) If $\|X-Y\|<\varepsilon$, then $-\varepsilon I<X-Y<\varepsilon I$.

(ii) Let $d=\alpha-\lambda_{S}$. If $d>0$, then $\alpha I-S \geq d I$.

Lemma 6. Suppose $\lim _{k \rightarrow \infty} S_{k}=S$ and $\lim _{k \rightarrow \infty} U_{k}=U$. Given an integer $n>0$ and a real number $\alpha>\lambda_{S}$, if $\lambda_{S}^{[n]}<$ $(>) \alpha$, there is an integer $N_{n}>0$ such that $\lambda_{P_{k}}^{[n]}<(>) \alpha$ for $k>N_{n}$.

Lemma 7. Suppose that $\left\{U_{k}\right\}$ is convergent. Let $\alpha>\lambda_{S}$ but $\alpha \notin\left\{\lambda_{S}^{[1]}, \lambda_{S}^{[2]}, \ldots\right\}$, and let $n_{\alpha}$ be the unique integer satisfying $\lambda_{S}^{\left[n_{\alpha}\right]}<\alpha<\lambda_{S}^{\left[n_{\alpha}+1\right]}$. Then there exists an integer $N>0$ such that for $\forall k>N$,

(i) $\lambda_{P_{k}}^{\left[n_{\alpha}\right]}<\alpha<\lambda_{P_{k}}^{\left[n_{\alpha}+1\right]}$.

(ii) $\mathbb{P}_{k}\left(\left\{\mathbf{P}_{k} \leq \alpha I\right\}\right)=1-\bar{\gamma}^{n_{\alpha}+1}$.

Proof of Theorem 1: From Proposition 6 (i), it follows that: 1) For the multi-variable case, when $X=S^{[m]}$ with $m \in$ $\left.\mathbb{Z}_{+}, \mathbb{P}_{\infty}(\{\mathbf{P}=X\}) \neq 0 ; 2\right)$ For the scalar case, $S^{[m]}=\lambda_{S}^{[m]}$, and thus when $x=\lambda_{S}^{[m]}$ with $m \in \mathbb{Z}_{+}, \mathbb{P}_{\infty}(\{\mathbf{P}=x\}) \neq$ 0 . Therefore, according to the definition of convergence in distribution, to avoid the possibility that $\mathbb{P}_{\infty}(\{\mathbf{P}=\alpha I\}) \neq 0$, we consider $\alpha \in\left(\lambda_{S}, \infty\right)$ but $\alpha \notin\left\{\lambda_{S}^{[1]}, \lambda_{S}^{[2]}, \ldots\right\}$.

It follows form Lemma 4(iii) and Proposition 6(ii) that for a given $\alpha>\lambda_{S}$, there exists an integer $n_{\alpha}$ such that $\lambda_{S}^{\left[n_{\alpha}\right]}<\alpha<\lambda_{S}^{\left[n_{\alpha}+1\right]}$ and $\mathbb{P}_{\infty}(\{\mathbf{P} \leq \alpha I\})=1-\bar{\gamma}^{n_{\alpha}+1}$. From Lemma 7, it follows that there exists an integer $N$ such that for $k>N, \mathbb{P}_{k}\left(\left\{\mathbf{P}_{k} \leq \alpha I\right\}\right)=1-\bar{\gamma}^{n_{\alpha}+1}=\mathbb{P}_{\infty}(\{\mathbf{P} \leq \alpha I\})$, which implies $\mathbb{P}_{k}\left(\left\{\mathbf{P}_{k} \leq \alpha I\right\}\right)$ converges to $\mathbb{P}_{\infty}(\{\mathbf{P} \leq \alpha I\})$. Therefore, according to Definition 2, we have $\mathbf{P}_{k} \stackrel{d}{\rightarrow} \mathbf{P}$.

\section{Stability of $\mathbb{E}\left[\mathbf{P}_{k}\right]$}

In this section, we deal with Problem 2. Before formulating the result, we give a lemma as follows.

Lemma 8. $\mathbb{E}\left[\mathbf{P}_{k}\right]=P_{k}^{[k]} \bar{\gamma}^{k}+\sum_{n=0}^{k-1} P_{k}^{[n]} \gamma \bar{\gamma}^{n}$.

Theorem 2. For the SS-UDP system in (1) with bounded inputs, under Assumption 1, if $\bar{\gamma}<\lambda_{A}^{-2}, \mathbb{E}\left[\mathbf{P}_{k}\right]$ is stable for $\forall P_{0} \geq 0$; if $\bar{\gamma}>\lambda_{A}^{-2}$, there exists a $P_{0}$ such that $\mathbb{E}\left[\mathbf{P}_{k}\right] \rightarrow \infty$.

Proof: Under Assumption 1, $S_{k}$ is convergent. Then it is bounded. Denote its bound by $S_{b}$. Let $\bar{U}$ be the bound for $U_{k}$. $\bar{U}$ exists by hypothesis. From (37), we have that for $0 \leq n \leq k$

$$
\begin{aligned}
P_{k}^{[n]} & \leq\left(\lambda_{A}^{2}\right)^{n} S_{b}+(\bar{U}+Q) \sum_{i=1}^{n}\left(\lambda_{A}^{2}\right)^{n-i} \\
& \leq\left(\lambda_{A}^{2}\right)^{n}\left(S_{b}+(\bar{U}+Q) /\left(\lambda_{A}^{2}-1\right)\right)=\left(\lambda_{A}^{2}\right)^{n} S^{*}
\end{aligned}
$$

where $S^{*} \triangleq S_{b}+(\bar{U}+Q) /\left(\lambda_{A}^{2}-1\right)$. By Lemma 8 ,

$$
\begin{aligned}
\mathbb{E}\left[\mathbf{P}_{k}\right] & =\sum_{n=0}^{k-1} P_{k}^{[n]} \gamma \bar{\gamma}^{n}+P_{k}^{[k]} \bar{\gamma}^{k} \\
& \leq \gamma S^{*} \sum_{n=0}^{k-1}\left(\bar{\gamma} \lambda_{A}^{2}\right)^{n}+S^{*}\left(\bar{\gamma} \lambda_{A}^{2}\right)^{k} \\
& \leq S^{*} \sum_{n=0}^{k}\left(\bar{\gamma} \lambda_{A}^{2}\right)^{n} \leq S^{*} /\left(1-\bar{\gamma} \lambda_{A}^{2}\right) .
\end{aligned}
$$

Therefore, for $\forall P_{0} \geq 0, \mathbb{E}\left[\mathbf{P}_{k}\right]$ is bounded.

From (37), we have $P_{k}^{[n]} \geq A^{n} S_{k-n}\left(A^{n}\right)^{\prime}$ for $0 \leq n \leq k$. Then $P_{k}^{[k]}>A^{k} S_{0}\left(A^{k}\right)^{\prime}$. From (20), it follows that $\mathbb{E}\left[\mathbf{P}_{k}\right]>$ $\bar{\gamma}^{k} P_{k}^{[k]}>\bar{\gamma}^{k} A^{k} P_{0}\left(A^{k}\right)^{\prime}$ where $S_{0}=P_{0}$. When $\lambda_{A}>1$, there is an eigenvalue $\lambda$ of $A$ such that $\lambda_{A}=|\lambda|$. Denote the corresponding eigenvector of $\lambda$ by $p \neq 0$. By letting $P_{0}=p p^{\prime}$, $\mathbb{E}\left[\mathbf{P}_{k}\right] \geq \bar{\gamma}^{k} A^{k} p p^{\prime}\left(A^{\prime}\right)^{k}=\bar{\gamma}^{k} \lambda^{k} p\left(\lambda^{k} p\right)^{\prime}=\left(\bar{\gamma} \lambda_{A}^{2}\right)^{k} P_{0}$. Hence, $\mathbb{E}\left[\mathbf{P}_{k}\right] \rightarrow \infty$ as $k \rightarrow \infty$. The proof is completed.

Remark 1. For the SS-UDP system, the stability of $\mathbb{E}\left[\mathbf{P}_{k}\right]$ depends on $\gamma$ but is independent of $\nu$. Similar phenomenon for the UDP-like system has been proved in [13].

\section{E. Estimation Performance Evaluation}

In this section, we focus on the estimation performance evaluation, but does not consider the design of controller. We adopt the metric $\mathbb{P}_{k}\left(\left\{\mathbf{P}_{k} \leq \alpha I\right\}\right)$ to evaluate the performance, as it gives a more complete characterization of the estimation performance $[29,30]$.

As previously shown, $\mathbf{P}_{k}$ depends on $\left\{u_{k}\right\}$, so does the computation of $\mathbb{P}_{k}\left(\left\{\mathbf{P}_{k} \leq \alpha I\right\}\right)$. However, in some cases, all the values of $\left\{u_{k}\right\}$ are not always known. For example, at the stage of the system design or before the running of system, designers sometimes do not know all the values of $\left\{u_{k}\right\}$ in advance, especially when $u_{k}$ is computed on-line, and only some partial information of the control sequence maybe available, such as its bound or limit. Therefore, we consider the following cases, in which $\left\{u_{k}\right\}$ is assumed to be bounded.

- Case 1: All the values of $\left\{u_{k}\right\}$ are known;

- Case 2: Only the upper bound $\bar{u}$ of $\left\{u_{k}\right\}$ is known;

- Case 3: $\left\{u_{k}\right\}$ is convergent and its limit $u_{\infty}$ is known.

Clearly, for case $2 U_{k}$ is bounded by $\bar{U}=\nu \bar{\nu} \bar{u}^{2} B B^{\prime}$, and for case $3 U_{k}$ converges to $U_{\infty}=\nu \bar{\nu} B u_{\infty} u_{\infty}^{\prime} B^{\prime}$.

Let $\Gamma_{k}(\alpha) \triangleq \mathbb{P}_{k}\left(\left\{\mathbf{P}_{k} \leq \alpha I\right\}\right)$.

When $\left\{u_{k}\right\}$ is convergent, Lemma 7 (ii) provides a way to compute $\Gamma_{k}(\alpha)$. However, it applies to the case that $\alpha \notin M_{S}$, where $M_{S} \triangleq\left\{\lambda_{S}^{[1]}, \lambda_{S}^{[2]}, \ldots\right\}$. When $\alpha \in M_{S}$, we can use $\Gamma_{k}(\alpha+\epsilon)$ to approximately calculate $\Gamma_{k}(\alpha)$, where we can choose $\epsilon$ to be a sufficiently small quantity, e.g., $10^{-5}$. In the following theorem, when Lemma 7 (ii) is used to compute $\Gamma_{k}(\alpha)$ and $\alpha \in M_{S}$, we assume that $\Gamma_{k}(\alpha)=\Gamma_{k}(\alpha+\epsilon)$.

Theorem 3. Given $\alpha>0$, for different cases, we have the following results for $\Gamma_{k}(\alpha)$ :

- For case $1, \Gamma_{k}(\alpha)$ can be computed by Algorithm 1.

- For case 2 , there is an integer $N$ such that $\Gamma_{k}(\alpha) \geq$ $1-\bar{\gamma}^{n_{b}+1} \triangleq p_{b d}$, for $k>N$, 
- For case 3, there is an integer $N$ such that $\Gamma_{k}(\alpha)=$ $1-\bar{\gamma}^{n_{c}+1} \triangleq p_{\text {conv }}$, for $k>N$,

where $n_{b}=n(\alpha, \bar{U})$ and $n_{c}=n\left(\alpha, U_{\infty}\right)$.

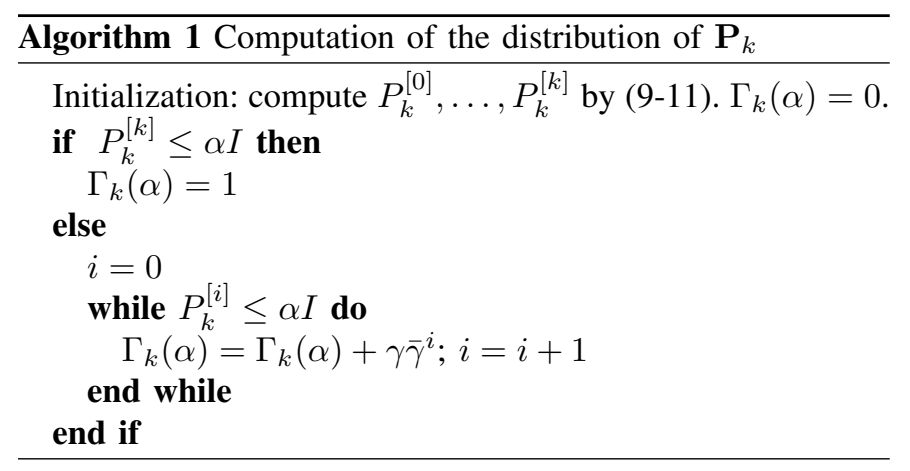

Proof: For case 1: If $0<\alpha I<P_{k}^{[0]}$, then $\alpha I<P_{k}^{[i]}$ for $0 \leq i \leq k$ due to the monotonicity of $P_{k}^{[i]}$. Consequently, $\Gamma_{k}(\alpha)=0$, which is equal to the result Algorithm 1 yields.

In the following, we consider the case $P_{k}^{[0]} \leq \alpha I$.

If $P_{k}^{[k]} \leq \alpha I$, then $\mathbf{P}_{k}(\theta) \leq \alpha I$ with $\theta \in F_{k}^{[i]}$ for $0 \leq i \leq k$. From Proposition 1 (ii), we have $\left\{\mathbf{P}_{k}<\alpha I\right\}=\Omega_{k}$. Then $\Gamma_{k}(\alpha)=\mathbb{P}_{k}\left(\left\{\mathbf{P}_{k}<\alpha I\right\}\right)=\mathbb{P}_{k}\left(\Omega_{k}\right)=1$.

Then we consider the case that $P_{k}^{[0]} \leq \alpha I<P_{k}^{[k]}$. Due to the monotonicity of $P_{k}^{[i]}$, it is clear that there exists an integer $0 \leq n_{\alpha}<k$ such that $P_{k}^{\left[n_{\alpha}\right]} \leq \alpha I$ and $P_{k}^{\left[n_{\alpha}+1\right]} \not \leq \alpha$. This integer $n_{\alpha}$ in fact is the largest integer $n$ such that $P_{k}^{[n]} \leq \alpha I$ holds. From Proposition 2, $\mathbf{P}_{k}(\theta)=P_{k}^{[i]}$ for $\theta \in F_{k}^{[i]}$. Therefore, $\mathbf{P}_{k}(\theta) \leq \alpha I$ if and only if $\theta \in \biguplus_{i=0}^{n_{\alpha}} F_{k}^{[i]}$, which implies $\left\{\mathbf{P}_{k} \leq \alpha I\right\}=\biguplus_{i=0}^{n_{\alpha}} F_{k}^{[i]} . \Gamma_{k}(\alpha)=\mathbb{P}_{k}\left(\left\{\mathbf{P}_{k} \leq\right.\right.$ $\alpha I\})=\mathbb{P}_{k}\left(\biguplus_{i=0}^{n_{\alpha}} F_{k}^{[i]}\right)=\sum_{i=0}^{n_{\alpha}} \mathbb{P}_{k}\left(F_{k}^{[i]}\right)=\sum_{i=0}^{n_{\alpha}} \gamma \bar{\gamma}^{i}$. Clearly, the routine in the while loop is to find out the $n_{\alpha}$ and then sum up $\gamma \bar{\gamma}^{i}$ to obtain $\sum_{i=0}^{n_{\alpha}} \gamma \bar{\gamma}^{i}$.

For case 2: Define a random variable $\overline{\mathbf{P}}_{k}$ on $\Omega_{k}$ by letting $U_{k}$ in $\psi_{k}$ be a constant quantity, i.e., $U_{k}=\bar{U}$. Then define $\bar{\psi}(P)=A P A^{\prime}+Q+\bar{U}$, and

$$
\bar{\Psi}^{[m]}(P)= \begin{cases}\underbrace{\bar{\psi} \circ \cdots \circ \bar{\psi}}_{m}(P), & \text { for } m=0 \\ \underbrace{}_{m} \text { for } 1 \leq m \leq k .\end{cases}
$$

Define $\bar{P}_{k}^{[i]} \triangleq \bar{\Psi}^{[i]}\left(S_{k-i}\right)$ and $\overline{\mathbf{P}}_{k}(\theta)=\bar{P}_{k}^{[i]}$ for $\theta \in F_{k}^{[i]}$.

Note that $\bar{\psi}^{[m]}(P) \geq \psi_{k}^{[m]}(P)$ for $\forall P \geq 0$. By comparing the definitions of $\bar{\Psi}^{[m]}(P)$ and $\Psi_{k}^{[m]}(P)$, it is easy to obtain that $\bar{\Psi}^{[m]}(P) \geq \Psi_{k}^{[m]}(P)$ for $\forall P \geq 0$. Thus, $\bar{P}_{k}^{[i]}=\bar{\Psi}^{[i]}\left(S_{k-i}\right) \geq \Psi_{k}^{[i]}\left(S_{k-i}\right)=P_{k}^{[i]}$. Then we have $\overline{\mathbf{P}}_{k}(\theta) \geq \mathbf{P}_{k}(\theta)$ for $\forall \theta \in \Omega_{k}$.

Let $u_{k} \equiv \bar{u}$. Obviously, $\left\{u_{k}\right\}$ is a convergent sequence. It follows from Lemma 7 (ii) that there is an integer $N$ such that for $k>N, \mathbb{P}_{k}\left(\left\{\overline{\mathbf{P}}_{k} \leq \alpha I\right\}\right)=1-\bar{\gamma}^{n_{b}+1}=p_{b d}$ where $n_{b}=n(\alpha, \bar{U})$. Since $\overline{\mathbf{P}}_{k}(\theta) \geq \mathbf{P}_{k}(\theta)$, we have $\left\{\overline{\mathbf{P}}_{k} \leq \alpha I\right\} \subset$ $\left\{\mathbf{P}_{k} \leq \alpha I\right\}$. Then $\mathbb{P}_{k}\left(\left\{\mathbf{P}_{k} \leq \alpha I\right\}\right) \geq \mathbb{P}_{k}\left(\left\{\overline{\mathbf{P}}_{k} \leq \alpha I\right\}\right)=p_{b d}$.

For case 3: The case 3 is the result of Lemma 7 (ii).

Estimation performance evaluation: For different cases, the results on the computation of $\Gamma_{k}(\alpha)$ are formulated in Theorem 3. For cases 2 and 3, without full knowledge of $\left\{u_{k}\right\}$,
Algorithm 1 does not work and the exact value of cannot be obtained. When $k$ is sufficiently large, $p_{b d}$ is a lower bound for $\Gamma_{k}(\alpha)$ and $p_{\text {conv }}$ is identical to $\Gamma_{k}(\alpha)$. They can be viewed as long term evaluations for estimation performance.

Remark 2. In Algorithm 1, at the time $k$, it requires to compute $k+1$ quantities, i.e., $P_{k}^{[0]}, \ldots, P_{k}^{[k]}$. The amounts of computation and PC memory increase linearly with time, which is not suitable for the system running over a long time. Hence, even when the full knowledge of $\left\{u_{k}\right\}$ is known, to avoid the time-consuming computation, one can use the results in cases 2 and 3 to obtain an approximation.

\section{OPTIMAL CONTROL}

In this section, we start with some useful lemmas, and then give answers to Problems 4 and 5 in Theorems 4 and 5 .

\section{A. Finite Horizon LQG control}

From (8), we have

$$
\mathbb{E}\left[\mathbf{P}_{k+1} \mid \mathcal{I}_{k}\right]=\bar{\gamma}\left(A \mathbf{P}_{k} A^{\prime}+Q+\bar{\nu} \nu B u_{k} u_{k}^{\prime} B^{\prime}\right)+\gamma S_{k+1} .
$$

Define the optimal value function

$$
\begin{aligned}
V_{N}\left(x_{N}\right) & =\mathbb{E}\left[x_{N}^{\prime} W_{N} x_{N} \mid \mathcal{I}_{N}\right] \\
V_{k}\left(x_{k}\right) & =\min _{u_{k}} \mathbb{E}\left[x_{k}^{\prime} W_{k} x_{k}+\nu_{k} u_{k}^{\prime} \Lambda_{k} u_{k}+V_{k+1}\left(x_{k+1}\right) \mid \mathcal{I}_{k}\right] .
\end{aligned}
$$

Lemma 9. $V_{k}\left(x_{k}\right)$ can be calculated as follows.

$$
V_{k}\left(x_{k}\right)=\mathbb{E}\left[x_{k}^{\prime} M_{k} x_{k} \mid \mathcal{I}_{k}\right]+\operatorname{tr}\left(\Delta_{k}\right)+\operatorname{tr}\left(H_{k} \mathbf{P}_{k}\right)
$$

where $H_{k}=T_{k}-M_{k}$,

$$
\begin{aligned}
M_{k}= & A^{\prime} M_{k+1} A+W_{k}-\nu A^{\prime} M_{k+1} B \\
& \times\left(\Lambda_{k}+B^{\prime}\left(\alpha M_{k+1}+\bar{\alpha} T_{k+1}\right) B\right)^{-1} B^{\prime} M_{k+1} A \\
T_{k}= & \gamma A^{\prime} M_{k+1} A+\bar{\gamma} A^{\prime} T_{k+1} A+W_{k} \\
\Delta_{k}= & \Delta_{k+1}+\left(\bar{\gamma} T_{k+1}+\gamma M_{k+1}\right) Q+\gamma H_{k+1} S_{k+1},
\end{aligned}
$$

with $M_{N}=T_{N}=W_{N}$ and $\Delta_{N}=0$.

Theorem 4. For the SS-UDP system, the optimal control minimizing $J_{N}$ is $u_{k}=L_{k} \hat{\mathbf{x}}_{k}$, where

$$
L_{k}=-\left(\Lambda_{k}+B^{\prime}\left(\alpha M_{k+1}+\bar{\alpha} T_{k+1}\right) B\right)^{-1} B^{\prime} M_{k+1} A
$$

and the corresponding cost function

$$
\begin{aligned}
J_{N}^{*}= & \mathbb{E}\left[x_{0}^{\prime} M_{0} x_{0}\right]+\operatorname{tr}\left(T_{0}-M_{0}\right) P_{0} \\
& +\sum_{j=1}^{N} \operatorname{tr}\left(\left(\bar{\gamma} T_{j}+\gamma M_{j}\right) Q+\gamma\left(T_{j}-M_{j}\right) S_{j}\right)
\end{aligned}
$$

where $M_{k}$ and $T_{k}$ are computed by (25).

Proof: From the derivation of $V_{k}\left(x_{k}\right)$ in Lemma 9, it is clear that $u_{k}=L_{k} \hat{\mathbf{x}}_{k}$ is the optimal control law minimizing the cost function. It follows from the dynamic programming theory that $J_{N}^{*}=V_{0}\left(x_{0}\right)$. Then (27) can be easily obtained by computing $V_{0}\left(x_{0}\right)$ via $(24)(25 \mathrm{c})$. The proof is completed.

Remark 3. It is known that the separation principle holds for the TCP-like system but fails for the UDP-like one [14]. For 
the SS-UDP system, the feedback gain $L_{k}$ can be computed offline by (25a)(25b)(26), but $\hat{\mathbf{x}}_{k}$ and $\mathbf{P}_{k}$ in (7) and (8) depend on $u_{k-1}$. Thus, the separation principle still does not hold.

\section{B. Infinite Horizon $L Q G$ control}

Let $W_{N}=W_{k}=W$ and $\Lambda_{k}=\Lambda$. Define two functions

$$
\begin{aligned}
\phi_{M}(M, T)= & A^{\prime} M A+W \\
& -\nu A^{\prime} M B\left(\Lambda+B^{\prime}(\alpha M+\bar{\alpha} T) B\right)^{-1} B^{\prime} M A \\
\phi_{T}(M, T)= & \bar{\gamma} A^{\prime} T A+\gamma A^{\prime} M A+W
\end{aligned}
$$

where $\bar{\alpha}=\bar{\gamma} \bar{\nu}$ and $\alpha=1-\bar{\alpha}$.

If $\lim _{N \rightarrow \infty} \frac{1}{N} J_{N}^{*}$ exists, so does the optimal control for the infinite horizon case. The existence of this limit depends on the convergences of $M_{k+1}=\phi_{M}\left(M_{k}, T_{k}\right)$ and $T_{k+1}=$ $\phi_{T}\left(M_{k}, T_{k}\right)$, which has been studied in Lemmas 8.2 and 8.3 of [14]. The result is formulated as follows.

Lemma 10 ([14]). There are $M_{\infty}>0$ and $T_{\infty}>0$ such that

$$
M_{\infty}=\phi_{M}\left(M_{\infty}, T_{\infty}\right) \text { and } T_{\infty}=\phi_{T}\left(M_{\infty}, T_{\infty}\right),
$$

if and only if $M_{k+1}=\phi_{M}\left(M_{k}, T_{k}\right)$ and $T_{k+1}=\phi_{T}\left(M_{k}, T_{k}\right)$ converge to $M_{\infty}$ and $T_{\infty}$, respectively.

Theorem 5. For the SS-UDP system, if there exist solutions $M_{\infty}>0$ and $T_{\infty}>0$ for (28), then

(i) the LQG control for infinite horizon case exists, i.e., $u_{k}=$ $L_{\infty} \hat{\mathbf{x}}_{k}$,

$$
L_{\infty}=\left(\Lambda+B^{\prime}\left(\alpha M_{\infty}+\bar{\alpha} T_{\infty}\right) B\right)^{-1} B^{\prime} M_{\infty} A,
$$

and

$$
J_{\infty}=\gamma \operatorname{tr}\left(T_{\infty}-M_{\infty}\right) S+\operatorname{tr}\left(\bar{\gamma} T_{\infty}+\gamma M_{\infty}\right) Q .
$$

(ii) The closed-loop system is mean square stable.

Proof of part (i): It follows from Lemma 10 that $M_{k}$ and $T_{k}$ converge, which implies the existence of the optimal control. $L_{\infty}$ and $J_{\infty}$ can be obtained by taking the limits of $L_{k}$ in (26) and $\frac{1}{N} J_{N}^{*}$ in (27).

Proof of part (ii): Let $e_{k}^{s}=x_{k}-\hat{\mathbf{x}}_{k}^{s}$. Note that under Assumption $1, \mathbb{E}\left[\left\|e_{k}^{s}\right\|^{2}\right]=\mathbb{E}\left[\left(e_{k}^{s}\right)^{\prime} e_{k}^{s}\right]=\operatorname{tr}\left(S_{k}\right)$ is convergent and then is bounded. Thus, if $\mathbb{E}\left[\left\|x_{k}\right\|^{2}\right]$ is bounded, so is $\mathbb{E}\left[\left\|\hat{\mathbf{x}}_{k}^{s}\right\|^{2}\right]$. Similarly, due to $x_{k}=\hat{\mathbf{x}}_{k}+e_{k}$, if $\mathbb{E}\left[\left\|\hat{\mathbf{x}}_{k}\right\|^{2}\right]$ and $\mathbb{E}\left[\left\|e_{k}\right\|^{2}\right]$ are bounded, then $\mathbb{E}\left[\left\|x_{k}\right\|^{2}\right]$ is bounded. Therefore, the MSS of the closed-loop system is studied by examining the boundedness of $\mathbb{E}\left[\left\|\hat{\mathbf{x}}_{k}\right\|^{2}\right]$ and $\mathbb{E}\left[\left\|e_{k}\right\|^{2}\right]$ instead of $\mathbb{E}\left[\left\|x_{k}\right\|^{2}\right]$, $\mathbb{E}\left[\left\|\hat{\mathbf{x}}_{k}\right\|^{2}\right]$, and $\mathbb{E}\left[\left\|\hat{\mathbf{x}}_{k}^{s}\right\|^{2}\right]$.

From (1) and (2), $x_{k+1}=A x_{k}+\nu_{k} B u_{k}+\omega_{k}$. Then,

$$
\begin{aligned}
\hat{\mathbf{x}}_{k+1}= & \bar{\gamma}_{k+1}\left(A \hat{\mathbf{x}}_{k}+\nu B u_{k}\right)+\gamma_{k+1} \hat{\mathbf{x}}_{k+1}^{s} \\
= & \left(A+\left(\bar{\gamma}_{k+1} \nu+\gamma_{k+1} \nu_{k}\right) B L\right) \hat{\mathbf{x}}_{k} \\
& +\gamma_{k+1} A e_{k}+\gamma_{k+1} \omega_{k}-\gamma_{k+1} e_{k+1}^{s} .
\end{aligned}
$$

From (7) and (30), by some algebraic derivations, we have

$$
\begin{aligned}
e_{k+1}= & \bar{\gamma}_{k+1}\left(A e_{k}+\left(\nu_{k}-\nu\right) B L \hat{\mathbf{x}}_{k}\right) \\
& +\bar{\gamma}_{k+1} \omega_{k}+\gamma_{k+1} e_{k+1}^{s} .
\end{aligned}
$$

Since $\mathbb{E}\left[\left\|e_{k+1}^{s}\right\|^{2}\right]$ and $\mathbb{E}\left[\left\|\omega_{k}\right\|^{2}\right]$ are bounded, it is pointed out in [31] that $\hat{\mathbf{x}}_{k+1}$ and $e_{k+1}$ in (30) and (31) are m.s. stable if and only if they are m.s. stable in the homogeneous part of (30) and (31) as follows.

$$
\begin{aligned}
\hat{\mathbf{x}}_{k+1} & =\left(A+\left(\bar{\gamma}_{k+1} \nu+\gamma_{k+1} \nu_{k}\right) B L\right) \hat{\mathbf{x}}_{k}+\gamma_{k+1} A e_{k} \\
e_{k+1} & =\bar{\gamma}_{k+1}\left(\nu_{k}-\nu\right) B L \hat{\mathbf{x}}_{k}+\bar{\gamma}_{k+1} A e_{k} .
\end{aligned}
$$

For brevity, the subscripts $\infty$ of $M_{\infty}$ and $T_{\infty}$ are removed. Denote the optimal control by $u_{k}^{*}$. Let $W_{k}=W$ and $\Lambda_{k}=\Lambda$ in (23). It follows from (23) that $V_{k}\left(x_{k}\right)=$ $\mathbb{E}\left[x_{k}^{\prime} W x_{k}+\nu_{k}\left(u_{k}^{*}\right)^{\prime} \Lambda u_{k}^{*}+V_{k+1}\left(x_{k+1}\right) \mid \mathcal{I}_{k}\right]$. By using the established property in [14] that

$$
\mathbb{E}\left[x_{k}^{\prime} M x_{k} \mid \mathcal{I}_{k}\right]=\hat{\mathbf{x}}_{k}^{\prime} M \hat{\mathbf{x}}_{k}+\operatorname{tr}\left(M \mathbf{P}_{k}\right),
$$

Eq. (24) can be rewritten as $V_{k}\left(x_{k}\right)=\hat{\mathbf{x}}_{k}^{\prime} M_{k} \hat{\mathbf{x}}_{k}+\operatorname{tr}\left(\Delta_{k}\right)+$ $\mathbb{E}\left[e_{k}^{\prime} T_{k} e_{k} \mid \mathcal{I}_{k}\right]$. By taking mathematical expectation over information $\mathcal{I}_{k}$, we have

$$
\begin{aligned}
& \mathbb{E}\left[V_{k+1}\left(x_{k+1}\right)-V_{k}\left(x_{k}\right)\right]=-\mathbb{E}\left[x_{k}^{\prime} W x_{k}+\nu\left(u_{k}^{*}\right)^{\prime} \Lambda u_{k}^{*}\right] \\
& \mathcal{V}_{k} \triangleq \mathbb{E}\left[\hat{\mathbf{x}}_{k}^{\prime} M_{k} \hat{\mathbf{x}}_{k}+e_{k}^{\prime} T_{k} e_{k}\right]=\mathbb{E}\left[V_{k}\left(x_{k}\right)\right]-\mathbb{E}\left[\operatorname{tr}\left(\Delta_{k}\right)\right] .
\end{aligned}
$$

From (25c), we have

$$
\begin{aligned}
\mathcal{V}_{k+1}-\mathcal{V}_{k}= & -\mathbb{E}\left[x_{k}^{\prime} W x_{k}+\nu\left(u_{k}^{*}\right)^{\prime} \Lambda u_{k}^{*}\right] \\
& +\left(\bar{\gamma} T_{k+1}+\gamma M_{k+1}\right) Q+\gamma H_{k+1} S_{k+1} .
\end{aligned}
$$

Observe that in Lemma 9, $x_{k}$ and $e_{k}$ are determined by (30) and (31). Here, what we consider is their homogeneous parts (32) and (33). Clearly, in the homogeneous parts there is no process noise $\omega_{k}$ and the estimation error $e_{k}^{s}$ is set to be zero as well, which is equivalent to setting their covariances to be zeros, that is, $Q=S_{k}=0$. Consequently, for the homogeneous parts,

$$
\begin{aligned}
\mathcal{V}_{k+1}-\mathcal{V}_{k} & =-\mathbb{E}\left[x_{k}^{\prime} W x_{k}+\nu\left(u_{k}^{*}\right)^{\prime} \Lambda u_{k}^{*}\right] \\
& =-\mathbb{E}\left[x_{k}^{\prime} W x_{k}+\nu \hat{\mathbf{x}}_{k}^{\prime} L^{\prime} \Lambda L \hat{\mathbf{x}}_{k}\right] \\
& =-\mathbb{E}\left[\hat{\mathbf{x}}_{k}^{\prime}\left(W+\nu L^{\prime} \Lambda L\right) \hat{\mathbf{x}}_{k}+e_{k}^{\prime} W e_{k}\right] .
\end{aligned}
$$

By repeatedly using the preceding equation, we have $\mathcal{V}_{k+1}-$ $\mathcal{V}_{0}=-\sum_{i=0}^{k} \mathbb{E}\left[\hat{\mathbf{x}}_{i}^{\prime}\left(W+\nu L^{\prime} \Lambda L\right) \hat{\mathbf{x}}_{i}+e_{i}^{\prime} W e_{i}\right]$. Since $\mathcal{V}_{k+1} \geq 0$,

$$
\mathcal{V}_{0} \geq \sum_{i=0}^{k} \mathbb{E}\left[\hat{\mathbf{x}}_{i}^{\prime}\left(W+\nu L^{\prime} \Lambda L\right) \hat{\mathbf{x}}_{i}+e_{i}^{\prime} W e_{i}\right] .
$$

Note that $\mathcal{V}_{0}=\mathbb{E}\left[\hat{\mathbf{x}}_{0}^{\prime} M_{0} \hat{\mathbf{x}}_{0}+e_{0}^{\prime} T_{0} e_{0}\right]$ is bounded and that $W+\nu L^{\prime} \Lambda L$ and $W$ are positive definite. The series in the right hand side of (35) is monotonically increasing and bounded so that it is convergent, which implies that $\mathbb{E}\left[\left\|\hat{\mathbf{x}}_{i}\right\|^{2}\right] \rightarrow 0$ and $\mathbb{E}\left[\left\|e_{i}\right\|^{2}\right] \rightarrow 0$ as $i \rightarrow \infty$. Then $\mathbb{E}\left[\left\|\hat{\mathbf{x}}_{k}\right\|^{2}\right]$ and $\mathbb{E}\left[\left\|e_{k}\right\|^{2}\right]$ are bounded. Thus, $\hat{\mathbf{x}}_{k+1}$ and $e_{k+1}$ are m.s. stable in the homogeneous part of (30) and (31), which implies the MMS of the closed-loop system.

\section{FurTher DisCuSSIONS ON CONDITIONS 1 AND 2}

As mentioned in Section I, for UDP-like systems, the theoretical analysis and practical use of the optimal estimation and control encounter intractable difficulties. In this section, we show that these difficulties remain if one of the Conditions 1 and 2 is not satisfied. Consider the following two cases.

Case I: Condition 1 holds, but Condition 2 is not satisfied. Even when the sensor has computational abilities, $\hat{\mathbf{x}}_{k}^{s}$ cannot 
be computed by the smart sensor, since its computation requires $u_{k}^{a}$, which can be seen from (4). What the smart sensor can send to the estimator is the raw measurement $y_{k}$. Hence, in this case, the system works like the conventional UDP-like system.

Case II: Condition 2 holds, but Condition 1 fails. Without computational abilities, the useful information the smart sensor can provide the estimator via the $\mathrm{S} / \mathrm{E}$ channel is $u_{k}^{a}$ and $y_{k}$. Then the estimator can recover the $\nu_{k}$ from $u_{k}^{a}$. However, $\left\{\nu_{k}\right\}$ is partially and stochastically recovered due to the losses of $u_{k}^{a}$ in the S/E channel. Therefore, this case can be viewed as the systems with ACK signals randomly lost. It is revealed in [11, 23 ] that it is computationally prohibitive to obtain the optimal estimator and LQG controller if ACK signals are randomly lost.

It can be concluded that if Conditions 1 and 2 are not satisfied simultaneously, solving the optimal estimation and control problems will confront with intractable difficulties.

\section{NUMERICAL EXAMPLES}

In this section, the main results we obtained are verified by some numerical examples. Consider the double inverted pendulum model in [43] with following parameters:

$$
\begin{aligned}
& A= \\
& {\left[\begin{array}{cccccc}
1 & -0.0004 & 0 & 0.0093 & 0 & 0 \\
0 & 1.0034 & -0.0010 & 0.0016 & 0.0090 & 0.0003 \\
0 & -0.0038 & 1.0032 & -0.0004 & 0.0008 & 0.0094 \\
0 & -0.0786 & 0.0063 & 0.8730 & 0.0083 & -0.0048 \\
0 & 0.6544 & -0.2380 & 0.3101 & 0.9034 & 0.0664 \\
0 & -0.7149 & 0.6137 & -0.0751 & 0.1579 & 0.8770
\end{array}\right]}
\end{aligned}
$$

$B=[0.0001 ;-0.0003 ; 0.0001 ; 0.0274 ;-0.0668 ; 0.0162]^{\prime}$ $C=\left[\begin{array}{cccccc}1 & 0 & 0 & 0 & 0 & 0 \\ 0 & 0 & 1 & 0 & 0 & 0 \\ 0 & 0 & 0 & 0 & 1 & 0\end{array}\right]$. Let $Q=0.01 I_{6}$ and $R=$ $0.01 I_{3}$, where $I_{3}$ and $I_{6}$ are identity matrices with dimensions 3 and 6 , respectively. $W=I_{6}$ and $\Lambda=2$.

1) Convergence of $\mathbf{P}_{k}$ : Let $\gamma=\nu=0.5$. Here, we do not draw the graphs of all $\Gamma_{k}(\alpha)$ to show $\Gamma_{k}(\alpha) \rightarrow \Gamma_{\infty}(\alpha)$, as their trajectories seriously overlap. Hence, we draw $\Gamma_{k}(\alpha)$ for some specific values $k=20,40,60,80$, and 100. As shown in Fig. 2 (a semi-log graph), $\Gamma_{k}(\alpha)$ converges to $\Gamma_{\infty}(\alpha)$, which implies the convergence of $\mathbf{P}_{k}$ to $\mathbf{P}$ in distribution.

2) Stability of $\mathbb{E}\left[\mathbf{P}_{k}\right]$ : The relationship between $\mathbb{E}\left[\mathbf{P}_{k}\right]$ and $\gamma$ is shown in Fig. 3. It can be seen that under different control inputs and packet arrival rate $\nu$, the stability condition for $\mathbb{E}\left[\mathbf{P}_{k}\right]$ is the same and is determined by the observation packet arrival rates. As claimed in Theorem 2 , for $\mathbb{E}\left[\mathbf{P}_{k}\right]$ to be stable, it requires $\bar{\gamma}<\lambda_{A}^{-2}$, i.e., $\gamma>0.0763$.

3) Estimation Performance Evaluation: Let $\gamma=\nu=0.5$. The exact values of $\Gamma_{k}(1)$ and $\Gamma_{k}(1.5)$ are calculated by Algorithm 1. Fig. 4(a) shows that if $\left\{u_{k}\right\}$ is bounded but not convergent, so is $\Gamma_{k}(1.5)$. When its bound $\bar{u}=15$ is known, by Theorem 3, the lower bound $p_{b d}$ for $\Gamma_{k}(1.5)$ can be obtained. It can be viewed as a lower bound for estimation performance. It can also be seen in Fig. 4(b) that if $\left\{u_{k}\right\}$ is convergent, so is $\Gamma_{k}(1)$, as claimed in Theorem 1. Based

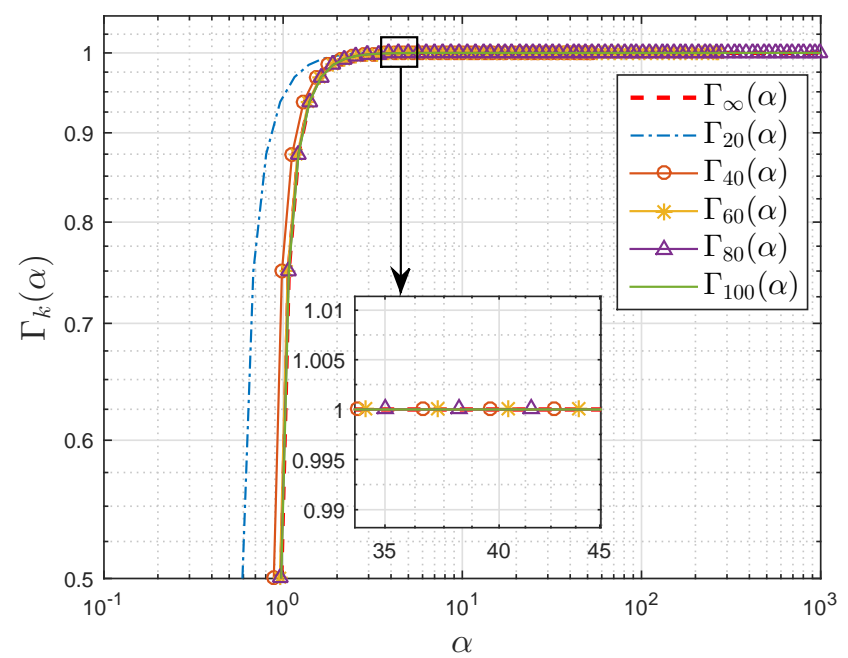

Fig. 2. Convergence of $\Gamma_{k}(\alpha)=\mathbb{P}_{k}\left(\left\{\mathbf{P}_{k} \leq \alpha\right\}\right)$

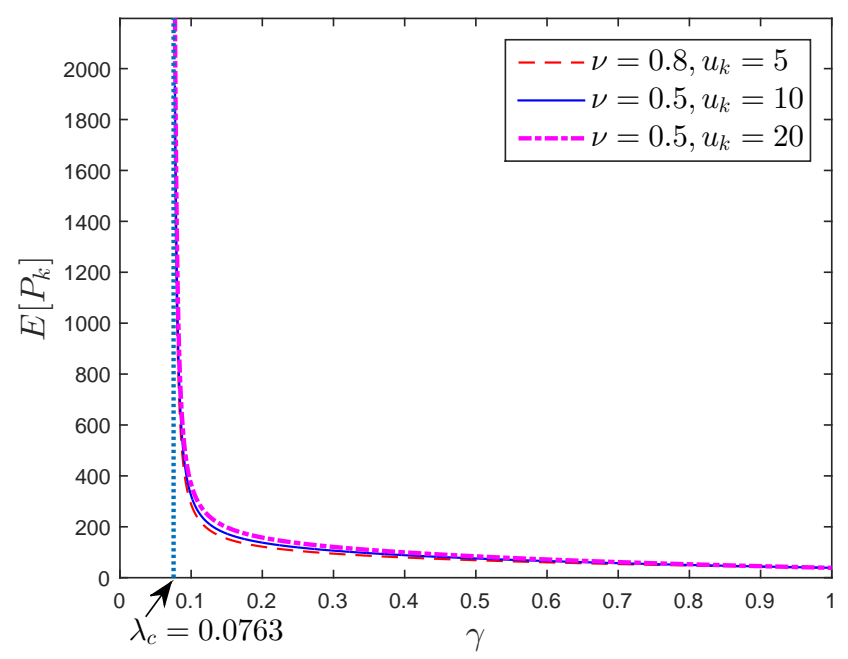

Fig. 3. Relationship between $\mathbb{E}\left[\mathbf{P}_{k}\right]$ and $\gamma$

on the limit of $\left\{u_{k}\right\}$, the quantity $p_{\text {conv }}$ can be computed by Theorem 3. When $k$ is sufficiently large, the exact value of $\Gamma_{k}(1)=p_{c o n v}$ is obtainable even when full knowledge of $\left\{u_{k}\right\}$ is unknown.

4) LQG control and MSS of the closed-loop system: Let $\gamma=\nu=0.8$. As shown in Fig. 5, it is easy to check the convergences of $M_{k}$ and $T_{k}$ by simulation. Then, it follows from Lemma 10 that there exist solutions $M_{\infty}>0$ and $T_{\infty}>0$ for (28), which guarantees the existence of the infinite LQG control. For the closed-loop SS-UDP system, we carry out 1000 times Monte Carlo simulations and then compute the mean values $\mathbb{E}\left[\left\|\hat{\mathbf{x}}_{k}\right\|\right]$ and $\mathbb{E}\left[\left\|e_{k}\right\|\right]$. As shown in Fig. $5, \mathbb{E}\left[\left\|\hat{\mathbf{x}}_{k}\right\|\right]$ and $\mathbb{E}\left[\left\|e_{k}\right\|\right]$ are bounded, which also implies the boundedness of $\mathbb{E}\left[\left\|\hat{\mathbf{x}}_{k}\right\|^{2}\right]$ and $\mathbb{E}\left[\left\|e_{k}\right\|^{2}\right]$. Note that $\mathbb{E}\left[\left\|e_{k}^{s}\right\|^{2}\right]=\operatorname{tr}\left(S_{k}\right)$ is bounded under Assumption 1. Thus, the closed-loop SS-UDP system is mean square stable, as claimed in Theorem 5. 


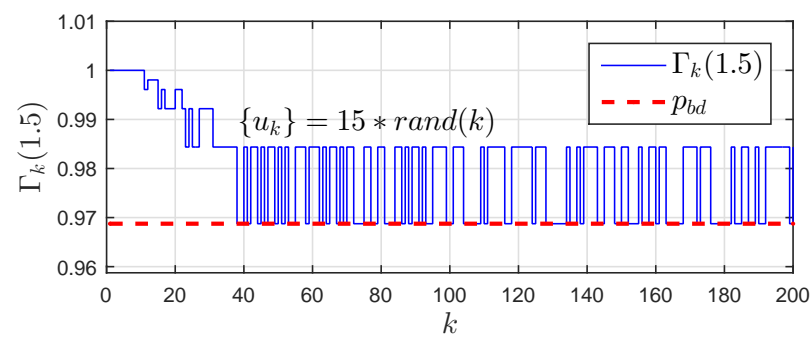

(a) The bounded but not convergent input case.

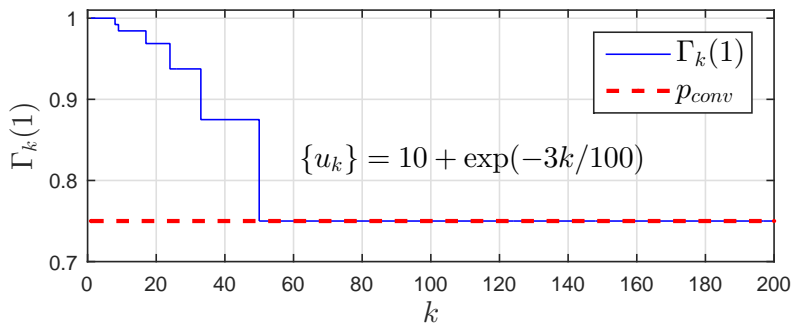

(b) The convergent input case.

Fig. 4. $\mathbb{P}_{k}\left(\left\{\mathbf{P}_{k}<I\right\}\right)$ and $\mathbb{P}_{k}\left(\left\{\mathbf{P}_{k}<1.5 I\right\}\right)$. The $\operatorname{rand}(k)$ stands for a sequence of i.i.d. Bernoulli random variables taking value in 0 or 1 .
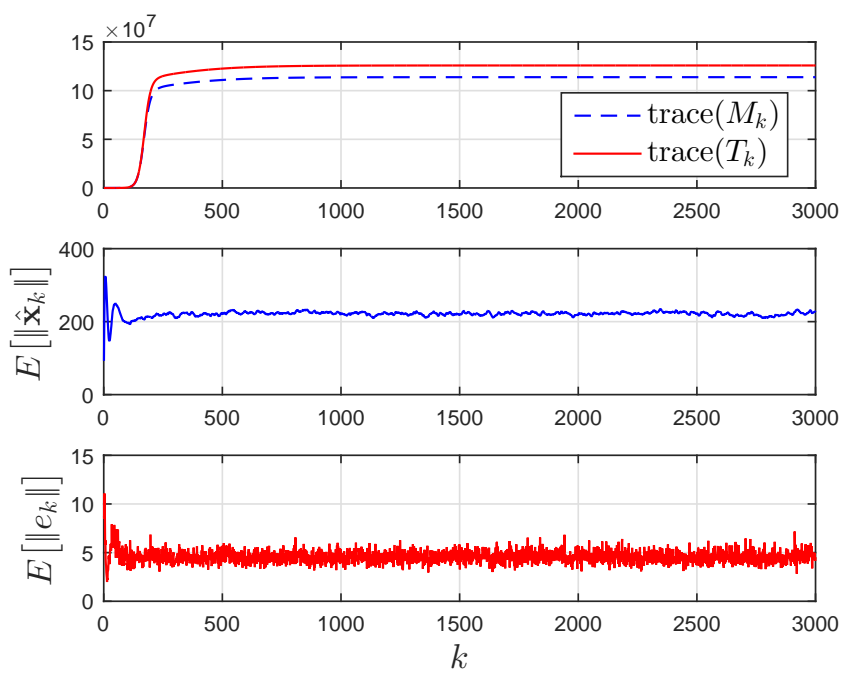

Fig. 5. Mean square stability of the closed-loop SS-UDP system

\section{CONCLUSION}

In this paper, we have shown that for conventional UDPlike systems there are some intrinsic difficulties to design the optimal estimation and LQG control. To overcome these difficulties, we have proposed two feasible conditions for the system structure. For UDP-like systems satisfying these two conditions, the optimal estimation has been derived. By constructing the probability spaces of the estimation error covariances, the stability condition for $\mathbb{E}\left[\mathbf{P}_{k}\right]$, the convergence of $\mathbf{P}_{k}$ in distribution, and some practical ways to evaluate the estimation performance have been established. The LQG controllers for both finite and infinite horizon cases have been developed. Examples are given to demonstrate the potential and effectiveness of the proposed new design techniques.
There are two possible extensions. One is to design eventtriggering conditions on the smart sensor side and then develop the corresponding event-based remote estimator, whose advantages [44] may help extend the results in this paper to UDPlike networks suffering from the limitation of communication and the restriction on the actuator/sensor power consumption. The other is to extend the obtained results for the single smart sensor node to the multiple-nodes case, and consider the distributed estimation and control problems [45] over UDPlike networks.

\section{APPENDIX}

Proof of Lemma 1: For the estimator, if $\gamma_{k}=1$, then the estimator receives $\hat{\mathbf{x}}_{k}^{s}$. Thus, $\hat{\mathbf{x}}_{k}=\hat{\mathbf{x}}_{k}^{s}$ and $\mathbf{P}_{k}=S_{k}$.

If $\gamma_{k}=0$, without $\hat{\mathbf{x}}_{k}^{s}$, the optimal estimate is in fact the prediction $\hat{\mathbf{x}}_{k}=A \hat{\mathbf{x}}_{k-1}+\nu B u_{k-1}$. Then, the prediction error covariance

$$
\begin{aligned}
\mathbf{P}_{k}= & \mathbb{E}\left[\left(x_{k}-\hat{\mathbf{x}}_{k}\right)\left(x_{k}-\hat{\mathbf{x}}_{k}\right)^{\prime} \mid \mathcal{I}_{k-1}\right] \\
= & \mathbb{E}\left[A\left(x_{k-1}-\hat{\mathbf{x}}_{k-1}\right)\left(x_{k-1}-\hat{\mathbf{x}}_{k-1}\right)^{\prime} A^{\prime} \mid \mathcal{I}_{k-1}\right] \\
& +\mathbb{E}\left[\omega_{k-1} \omega_{k-1}^{\prime}\right]+\mathbb{E}\left[\left(\nu_{k}-\nu\right)^{2}\right] B u_{k-1} u_{k-1}^{\prime} B^{\prime} \\
= & A \mathbf{P}_{k-1} A^{\prime}+Q+U_{k-1} .
\end{aligned}
$$

Proof of Proposition 1: Proof of part (i): Without loss of generality, we assume that $i<j$. By definition, for each $\theta_{a} \in F_{k}^{[i]}$, the element in the $i+1$ th position of $\theta_{a}$ is 1 . According to the definition of $F_{k}^{[j]}$, for each $\theta_{b} \in F_{k}^{[j]}$, from left to right, the first 1 occurs in the $j+1$ th position. It means that the element in the $i+1$ th position of $\theta_{b}$ must be 0 due to $i+1<j+1$. Therefore, $\theta_{a} \notin F_{k}^{[j]}$, vice versa. Part (i) is proved.

Proof of part (ii): It is clear that $F_{k}^{[i]} \subseteq \Omega_{k}$. Thus, we have $\biguplus_{i=0}^{k} F_{k}^{[i]} \subseteq \Omega_{k}$. In the following, we show that $\biguplus_{i=0}^{k} F_{k}^{[i]} \supseteq$ $\Omega_{k}$. For each $\theta \in \Omega_{k}$, if all the elements in $\theta$ are 0 , then by definition $\theta \in F_{k}^{[k]}$. If not, there must be at least one 1 in $\theta$. From left to right, denote the position where the first 1 occurs by $i+1$. According to the definition of $F_{k}^{[i]}, \theta \in F_{k}^{[i]}$. Thus, we have $\theta \in F_{k}^{[i]}$ for $\forall \theta \in \Omega_{k}$, which means $\biguplus_{i=0}^{k} F_{k}^{[i]} \supseteq \Omega_{k}$. Part (ii) is proved.

Proof of part (iii): By definition, $F_{k}^{[j]}$ consists of points of the form $\theta=\left(0, \ldots, 0, \gamma_{k-j}=1, \times, \ldots, \times\right)$. For each $\theta \in F_{k}^{[j]}, \mathbb{P}_{k}(\{\theta\})=\bar{\gamma}^{j} \gamma \mathbb{P}_{\gamma}\left(\gamma_{k-j-1}\right) \cdots \mathbb{P}_{\gamma}\left(\gamma_{1}\right)$.

According to the definition of $\mathbb{P}_{k}(A)$,

$$
\begin{aligned}
& \mathbb{P}_{k}\left(F_{k}^{[j]}\right)=\sum_{\theta \in F_{k}^{[j]}} \mathbb{P}_{k}(\{\theta\})=\sum_{\gamma_{k-j-1}=0}^{1} \cdots \sum_{\gamma_{1}=0}^{1} \mathbb{P}_{k}(\{\theta\}) \\
= & \sum_{\gamma_{k-j-1}=0}^{1} \cdots \sum_{\gamma_{1}=0}^{1} \gamma \bar{\gamma}^{j} \mathbb{P}_{\gamma}\left(\gamma_{k-j-1}\right) \cdots \mathbb{P}_{\gamma}\left(\gamma_{1}\right) \\
= & \gamma \bar{\gamma}^{j}\left(\sum_{\gamma_{k-j-1}=0}^{1} \mathbb{P}_{\gamma}\left(\gamma_{k-j-1}\right)\right) \cdots\left(\sum_{\gamma_{1}=0}^{1} \mathbb{P}_{\gamma}\left(\gamma_{1}\right)\right)=\gamma \bar{\gamma}^{j}
\end{aligned}
$$

where the last line is obtained by noting that $\sum_{\gamma_{i}=0}^{1} \mathbb{P}_{\gamma}\left(\gamma_{i}\right)=$ 1 , for $1 \leq i \leq k$. For $F_{k}^{[k]}, \mathbb{P}_{k}\left(F_{k}^{[k]}\right)=\mathbb{P}_{k}(\{(0, \ldots, 0)\})=$ $\bar{\gamma}^{k}$, which completes the proof. 
Proof of Lemma 2: By (5) and (6),

$$
\begin{aligned}
S_{k-n} & =\bar{S}_{k-n}-K_{k-n} C^{\prime} \bar{S}_{k-n} \\
& \leq \bar{S}_{k-n}=A S_{k-n-1} A^{\prime}+Q \\
& \leq A S_{k-n-1} A^{\prime}+Q+U_{k-n-1} \\
& =\psi_{k-n-1}\left(S_{k-n-1}\right) .
\end{aligned}
$$

From the definition of $\Phi_{k}^{[n]}$, it is clear that if $P_{1} \leq P_{2}$, then $\Phi_{k}^{[n]}\left(P_{1}\right) \leq \Phi_{k}^{[n]}\left(P_{2}\right) . P_{k}^{[n+1]}=\Phi_{k}^{[n+1]}\left(S_{k-n-1}\right)=$ $\Phi_{k}^{[n]}\left(\psi_{k-n-1}\left(S_{k-n-1}\right)\right) \geq \Phi_{k}^{[n]}\left(S_{k-n}\right)=P_{k}^{[n]}$.

Proof of Proposition 2: For each $\theta \in F_{k}^{[j]}$, it takes the form that $\theta=\left\{\gamma_{k}=0, \ldots, \gamma_{k-j+1}=0, \gamma_{k-j}=\right.$ $1, \times, \ldots, \times\}$. From (8), it follows that if $\gamma_{k-j}=1$, then $\mathbf{P}_{k-j}=S_{k-j}$ no matter what values $\left\{\gamma_{k-j-1}, \ldots, \gamma_{1}\right\}$ take. Due to $\left\{\gamma_{k}=0, \ldots, \gamma_{k-j+1}=0\right\}$, it follows from (8) that $\mathbf{P}_{k}=\psi_{k-1} \circ \cdots \circ \psi_{k-j}\left(S_{k-j}\right)=\Psi_{k}^{[j]}\left(S_{k-j}\right)=P_{k}^{[j]}$.

Proof of Proposition 3: Parts (i) and (ii) can be easily proved by following the similar line of argument in the proofs of parts (i) and (ii) of Proposition 1. Thus, the proofs of (i) and (ii) is not presented here for saving space.

We prove part (iii) by contradiction. It is no loss of generality to consider $B$ is a union of countable sets. Suppose that the representation of $B$ is not unique, then $B=\biguplus_{i \in \mathcal{N}_{B_{1}}} A^{[i]}=$ $\biguplus_{i \in \mathcal{N}_{B_{2}}} A^{[i]}$, where $\mathcal{N}_{B_{1}}=\left\{n_{1}, n_{2}, \ldots\right\} \biguplus\left\{n_{1}^{a}, n_{2}^{a}, \ldots\right\}$, $\mathcal{N}_{B_{2}}=\left\{n_{1}, n_{2}, \ldots\right\} \biguplus\left\{n_{1}^{b}, n_{2}^{b}, \ldots\right\},\left\{n_{1}, n_{2}, \ldots\right\}$ is the common set in $\mathcal{N}_{B_{1}}$ and $\mathcal{N}_{B_{2}}$, and $\left\{n_{1}^{a}, n_{2}^{a}, \ldots\right\} \cap\left\{n_{1}^{b}, n_{2}^{b}, \ldots\right\}=$ $\emptyset$. For $\forall n_{i}^{a}$ with $i \geq 1$, let $\theta \in A^{\left[n_{i}^{a}\right]}$. From part (i), it follows that $\theta \notin A^{\left[n_{j}\right]}$ due to $n_{j} \neq n_{i}^{a}$ for $j \geq 1$, and that $\theta \notin A^{\left[n_{j}^{b}\right]}$ due to $n_{i}^{a} \neq n_{j}^{b}$ for $\forall i$ and $j$. Therefore, $\theta \notin\left(\biguplus_{j \in\left\{n_{1}, n_{2}, \ldots\right\}} A^{[j]}\right) \biguplus\left(\biguplus_{j \in\left\{n_{1}^{b}, n_{2}^{b}, \ldots\right\}} A^{[j]}\right)=$ $\biguplus_{i \in \mathcal{N}_{B_{2}}} A^{[i]}=B$, which is a contradiction to $\theta \in n_{i}^{a} \subseteq B$. Thus, the presentation of $B$ is unique.

Let $B_{i}=\biguplus_{k \in \mathcal{N}_{B_{i}}} A^{[k]}$ and $B_{j}=\biguplus_{k \in \mathcal{N}_{B_{j}}} A^{[k]}$. For any $n_{i} \in \mathcal{N}_{B_{i}}, A^{\left[n_{i}\right]} \subseteq B_{i}$ but $A^{\left[n_{i}\right]} \nsubseteq B_{j}$ due to the hypothesis that $B_{i}$ and $B_{j}$ are disjoint, which implies $n_{i} \notin \mathcal{N}_{B_{j}}$. Similarly, we can obtain that for any $n_{j} \in \mathcal{N}_{B_{j}}, n_{j} \notin \mathcal{N}_{B_{i}}$, which implies $\mathcal{N}_{B_{i}} \cap \mathcal{N}_{B_{j}}=\emptyset$. Then, it is straightforward to have $B_{i} \biguplus B_{j}=\left(\biguplus_{k \in \mathcal{N}_{B_{i}}} A^{[k]}\right) \biguplus\left(\biguplus_{k \in \mathcal{N}_{B_{j}}} A^{[k]}\right)=$ $\biguplus_{k \in \mathcal{N}_{B_{i}} \biguplus \mathcal{N}_{B_{j}}} A^{[k]}$.

Proof of part (iv): For $\forall \theta \in \biguplus_{i=1}^{\infty} B_{i}$, there exists an unique integer $i$ such that $\theta \in B_{i}$, and then there exists an unique integer $j \in \mathcal{N}_{B_{i}}$ such that $\theta \in A^{[j]}$. Thus, $\theta \in A^{[j]} \subseteq \biguplus_{k \in \biguplus_{i=1}^{\infty} \mathcal{N}_{B_{i}}} A^{[k]}$, due to $j \in \biguplus_{i=1}^{\infty} \mathcal{N}_{B_{i}}$. Then we have $\biguplus_{i=1}^{\infty} B_{i} \subseteq \biguplus_{k \in \biguplus_{i=1}^{\infty} \mathcal{N}_{B_{i}}} A^{[k]}$. It is clear that for any

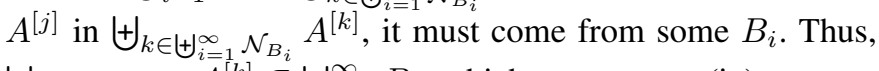

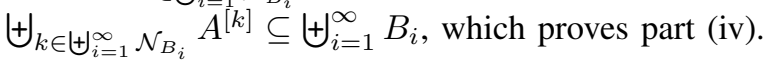

Proof of Proposition 4: Proof of part (i): The three conditions (i-iii) in Definition 5 for a collection of subsets to be a field are checked for $\mathscr{A}$. (i) By definition of $\mathscr{A}, \Omega_{\infty} \in \mathscr{A}$. (ii) If $B \in \mathscr{A}$, then there exists a finite or countable subset $\mathcal{N}_{B} \subseteq \overline{\mathbb{Z}}_{+}$such that $B=\biguplus_{i \in \mathcal{N}_{B}} A^{[i]}$. Note $\mathcal{N}_{B}{ }^{c} \subseteq \overline{\mathbb{Z}}_{+}$is a finite or countable subset as well. It follows from Proposition 3(ii) that $B^{c}=\biguplus_{i \in \mathcal{N}_{B}{ }^{c}} A^{[i]} \in \mathscr{A}$. (iii) Let $B_{1}, \ldots, B_{n} \in \mathscr{A}$. Then $\bigcup_{i=1}^{n} B_{i}=\bigcup_{i=1}^{n} \biguplus_{j \in \mathcal{N}_{B_{i}}} A^{[j]}=\biguplus_{j \in \mathcal{N}_{B}} A^{[j]}$, where
$\mathcal{N}_{B}=\bigcup_{i=1}^{n} \mathcal{N}_{B_{i}} \cdot \mathcal{N}_{B} \subseteq \overline{\mathbb{Z}}_{+}$is a finite or countable set. Thus, $\bigcup_{i=1}^{n} B_{i} \in \mathscr{A}$. Therefore, $\mathscr{A}$ is a field.

Proof of part (ii): Let $\left\{B_{i}\right\}$ be a countable collection of mutually disjoint subsets of $\mathscr{A}$. Denote $B=\biguplus_{i=1}^{\infty} B_{i}$. From Proposition 3(iv), $B=\biguplus_{k \in \biguplus_{i=1}^{\infty} \mathcal{N}_{B_{i}}} A^{[k]}$. Let $\mathcal{N}_{B}=$ $\biguplus_{i=1}^{\infty} \mathcal{N}_{B_{i}}$. By (14),

$$
\begin{aligned}
\mathbb{P}^{*}(B) & =\sum_{k \in \mathcal{N}_{B}} p_{k}=\sum_{k \in \biguplus_{i=1}^{\infty} \mathcal{N}_{B_{i}}} p_{k} \\
& =\sum_{k \in\left\{\mathcal{N}_{B_{1}}, \mathcal{N}_{B_{2}}, \ldots\right\}} p_{k} \\
& =\sum_{i=1}^{\infty}\left(\sum_{k \in \mathcal{N}_{B_{i}}} p_{k}\right)=\sum_{i=1}^{\infty} \mathbb{P}^{*}\left(B_{i}\right) .
\end{aligned}
$$

By definition, $\mathbb{P}^{*}$ is a nonnegative countably additive set function on the field $\mathscr{A}$, and thus it is a measure on the field $\mathscr{A}$, which proves part (ii).

Proof of part (iii): As $\mathbb{P}^{*}$ is countably additive, it naturally is finitely additive. According to the definition of $\sigma$-finite, part (iii) can be readily proved by noting the facts that $\Omega_{\infty}=$ $\biguplus_{i \in \overline{\mathbb{Z}}_{+}}^{\infty} A^{[i]}$ (Proposition 3), and that $\mathbb{P}^{*}\left(A^{[i]}\right)<1, i \in \overline{\mathbb{Z}}_{+}$.

Proof of Proposition 5: It has been proved that $\mathbb{P}^{*}$ and $\mathscr{A}$ satisfy the conditions required in Lemma 3. From Lemma 3 , it follows that there exists a probability measure $\mathbb{P}_{\infty}$ on $\mathscr{F}_{\infty}$ such that $\mathbb{P}_{\infty}(A)=\mathbb{P}^{*}(A)$ for $\forall A \in \mathscr{A}$. By noting that $\mathscr{A}_{0} \subseteq \mathscr{A}$, Proposition 5 is proved.

Proof of Lemma 4: Proof of part (i): From (15), (16), and (17), by some algebraic computations, we have

$$
S^{[n]}=A^{n} S\left(A^{n}\right)^{\prime}+\sum_{i=1}^{n} A^{n-i}(U+Q)\left(A^{n-i}\right)^{\prime} .
$$

From the definitions of $\psi$ and $\psi_{k}$, it follows that 1) if $P_{1}>P_{2}$, then $\psi\left(P_{1}\right)>\psi\left(P_{2}\right)$ and $\psi_{k}\left(P_{1}\right)>\psi_{k}\left(P_{2}\right)$; 2) $S^{[k+1]}=$ $\Psi^{[k+1]}(S)=\psi\left(\Psi^{[k]}(S)\right)=\psi\left(S^{[k]}\right)$. Note that $S^{[0]}=S=$ $\bar{S}-\bar{S} C^{\prime}\left(C \bar{S} C^{\prime}+R\right)^{-1} C \bar{S} \leq \bar{S}=A S A^{\prime}+Q \leq A S A^{\prime}+$ $Q+U=\psi(S)=S^{[1]}$. Then $\lambda_{S}^{[0]} \leq \lambda_{S}^{[1]}$. Part (i) holds for $n=0$. Suppose that part (i) holds for $n=1, \ldots, k$. We check the case $n=k+1 . S^{[k+1]}=\psi\left(S^{[k]}\right) \geq \psi\left(S^{[k-1]}\right)=S^{[k]}$. Hence, $\left\{S^{[n]}\right\}$ is an increasing sequence.

To prove that $\left\{S^{[n]}\right\}$ strictly increases, we show that $S^{[n+1]} \neq S^{[n]}$ for $\forall n \in \mathbb{Z}_{+}$. We have $S^{[n]}>0$ for $\forall n \in \mathbb{Z}_{+}$ due to $S>0$. If there is an integer $k \in \mathbb{Z}_{+}$such that $S^{[k+1]}=S^{[k]}$, then from (15)-(17) we have $S^{[k]}=S^{[k+1]}=$ $A S^{[k]} A^{\prime}+Q+U$. However, it is well known that for $\lambda_{A}>1$ there is no positive define solution $P$ for $P=A P A^{\prime}+Q+U$. Hence, $S^{[k+1]} \neq S^{[k]}$ for $\forall n \in \mathbb{Z}_{+}$. Part (i) is proved.

Proof of part (ii): $\lambda_{S}^{[k]} \leq \lambda_{S}^{[k+1]}$ is an immediate result of part (i). From (36), $S^{[n]} \geq A^{n} S\left(A^{n}\right)^{\prime} \geq \underline{\lambda}_{S}\left(A A^{\prime}\right)^{n}$. Thus, $\lambda_{S}^{[n]} \geq \underline{\lambda}_{S}\left(\lambda_{A}\right)^{2 n}$. Due to the assumption that $\lambda_{A}>1$, we have $\lim _{n \rightarrow \infty} \lambda_{S}^{[n]}=\infty$.

Proof of part (iii): The existence and uniqueness of such $n_{\alpha}$ are evident, due to the monotonicity of $\lambda_{S}^{[n]}$ and the property that $\lambda_{S}^{[n]} \rightarrow \infty$ as $n \rightarrow \infty$.

When $A$ and $Q$ is fixed, $n_{\alpha}$ only depends on $\alpha$ and the $U$ in $\psi(\cdot)$, and is also uniquely determined by $\alpha$ and $U$. Thus, the function $n_{\alpha}=n(\alpha, U)$ is well-defined. 
Proof of Proposition 6: Proof of part (i):From (18), it follows that $\mathbf{P}(\theta)=0$ only when $\theta \in A^{[\infty]}$. Thus, $\{\mathbf{P}(\theta)=0\}=$ $A^{[\infty]}$. By Proposition 5 and (13), $\mathbb{P}_{\infty}\left(A^{[\infty]}\right)=\mathbb{P}^{*}\left(A^{[\infty]}\right)=0$. By Lemma 4(i) and the definition of $\mathbf{P}(\theta)$ in (18), it is clear that $\mathbf{P}(\theta)=S^{[m]}$ only when $\theta \in A^{[m]}$. Thus, $\{\mathbf{P}(\theta)=$ $\left.S^{[m]}\right\}=A^{[m]}$. Then it follows from Proposition 5 and (13) that $\mathbb{P}_{\infty}\left(\left\{\mathbf{P}(\theta)=S^{[m]}\right\}\right)=\mathbb{P}_{\infty}\left(A^{[m]}\right)=\mathbb{P}^{*}\left(A^{[m]}\right)=\gamma \bar{\gamma}^{m}$.

Proof of part (ii): According to the definition of $\mathbf{P}$, it may take the values $\left\{0, S, S^{[1]}, \ldots\right\}$. Note that $\lambda_{S}^{[n]} \leq \alpha$ is equivalent to $S^{[n]} \leq \alpha I$. It follows from Lemma 4(i) and (iii) that for a given $\alpha, S^{[i]} \leq \alpha I$ for $0 \leq i \leq n_{\alpha}$, where $n_{\alpha}=n(\alpha, U)$. By the definition of $\mathbf{P},\{\mathbf{P} \leq$ $\alpha I\}$ consists of $\left\{A^{[\infty]}, A^{[0]}, \ldots, A^{\left[n_{\alpha}\right]}\right\}$. That is, $\{\mathbf{P} \leq$ $\alpha I\}=A^{[\infty]} \biguplus\left(\biguplus_{i=0}^{n_{\alpha}} A^{[i]}\right)$. Then $\mathbb{P}_{\infty}\left(A^{[\infty]} \biguplus\left(\biguplus_{i=0}^{n_{\alpha}} A^{[i]}\right)\right)=$ $\mathbb{P}_{\infty}\left(A^{[\infty]}\right)+\sum_{i=0}^{n_{\alpha}} \mathbb{P}_{\infty}\left(A^{[i]}\right)=\sum_{i=0}^{n_{\alpha}} \gamma \bar{\gamma}^{i}=1-\bar{\gamma}^{n_{\alpha}+1}$.

Proof of Lemma 5: Part (i) can be proved by noting the fact if $\|M\|<\varepsilon$ then $-\varepsilon<M<\varepsilon$, and part (ii) is proved by noting that $(\alpha-d) I=\lambda_{S} I \geq S$.

Proof of Lemma 6: From (9), (10), and (11), we have

$$
\begin{aligned}
P_{k}^{[n]}= & \Psi_{k}^{[n]}\left(S_{k-n}\right) \\
= & A^{n} S_{k-n}\left(A^{n}\right)^{\prime}+A^{n-1}\left(U_{k-1}+Q\right)\left(A^{n-1}\right)^{\prime} \\
& +A^{n-2}\left(U_{k-2}+Q\right)\left(A^{n-2}\right)^{\prime}+\ldots+\left(U_{k-n}+Q\right) \\
= & A^{n} S_{k-n}\left(A^{n}\right)^{\prime}+\sum_{i=1}^{n} A^{n-i}\left(U_{k-i}+Q\right)\left(A^{n-i}\right)^{\prime} .
\end{aligned}
$$

Due to the convergence of $\left\{S_{k}\right\}$ and $\left\{U_{k}\right\}$, for $\forall \varepsilon_{S}>0$ and $\forall \varepsilon_{U}>0$, there exists an integer $N_{n} \in \mathbb{N}$ such that $\| S_{k-n}-$ $S \|<\varepsilon_{S}$ and $\left\|U_{k-n}-U\right\|<\varepsilon_{U}$, for $k>N_{n}$. By (36),

$$
\begin{aligned}
& \left\|P_{k}^{[n]}-S^{[n]}\right\| \\
\leq & \left\|A^{n}\left(S_{k-n}-S\right)\left(A^{n}\right)^{\prime}+\sum_{i=1}^{n} A^{n-i}\left(U_{k-i}-U\right)\left(A^{n-i}\right)^{\prime}\right\| \\
\leq & \varepsilon_{S}\left\|A^{n}\left(A^{n}\right)^{\prime}\right\|+\varepsilon_{U} \sum_{i=0}^{n-1}\left\|A^{i}\left(A^{i}\right)^{\prime}\right\| .
\end{aligned}
$$

Let $d=\left|\alpha-\lambda_{S}^{[n]}\right|$. For a given $\varepsilon$ with $0<\varepsilon<d$, we can obtain $\left\|P_{k}^{[n]}-S^{[n]}\right\|<\varepsilon$ for $k>N_{n}$ by choosing sufficiently small $\varepsilon_{S}$ and $\varepsilon_{U}$. By Lemma 5 (i), we have $-\varepsilon I<S^{[n]}-P_{k}^{[n]}<\varepsilon I$, $\forall k>N_{n}$.

Proof of $\left(\lambda_{S}^{[n]}<\alpha\right) \Rightarrow\left(\lambda_{P_{k}}^{[n]}<\alpha\right)$ : By using Lemma 5 (ii), $\alpha I-P_{k}^{[n]}=\left(\alpha I-S^{[n]}\right)+\left(S^{[n]}-P_{k}^{[n]}\right)>d I-\varepsilon I>0$. Hence, $P_{k}^{[n]}<\alpha I$, which implies $\lambda_{P_{k}}^{[n]}<\alpha$.

Proof of $\left(\lambda_{S}^{[n]}>\alpha\right) \Rightarrow\left(\lambda_{P_{k}}^{[n]}>\alpha\right): S^{[n]}-P_{k}^{[n]}<\varepsilon I \Rightarrow$ $S^{[n]}<\varepsilon I+P_{k}^{[n]} \Rightarrow \lambda_{S}^{[n]}<\varepsilon+\lambda_{P_{k}}^{[n]} \Rightarrow \lambda_{P_{k}}^{[n]}-\lambda_{S}^{[n]}>-\varepsilon$. Then $\lambda_{P_{k}}^{[n]}-\alpha=\left(\lambda_{P_{k}}^{[n]}-\lambda_{S}^{[n]}\right)+\left(\lambda_{S}^{[n]}-\alpha\right)>-\varepsilon+d>0$.

Proof of Lemma 7: Proof of part (i): From Lemma 6, we have $\lambda_{P_{k}}^{\left[n_{\alpha}\right]}<\alpha$ for $k>N_{n_{\alpha}}$ and $\alpha<\lambda_{P_{k}}^{\left[n_{\alpha}+1\right]}$ for $k>N_{n_{\alpha}+1}$. By letting $N=\max \left(N_{n_{\alpha}}, N_{n_{\alpha}+1}\right)$, part (i) is proved.

Proof of part (ii). From part (i) and the monotonicity of $P_{k}^{[n]}$ (Lemma 2), it follows that for $k>N, \mathbf{P}_{k} \leq \alpha I$ if and only if $\mathbf{P}_{k}$ takes the value within $\left\{P_{k}^{[0]}, \ldots, P_{k}^{\left[n_{\alpha}\right]}\right\}$. It is shown in Proposition 2 that $\mathbf{P}_{k}(\theta)=P_{k}^{[i]}$ for $\theta \in F_{k}^{[i]}$. Therefore, $\left\{\mathbf{P}_{k} \leq \alpha I\right\}$ consists of $\left\{F_{k}^{[0]}, \ldots, F_{k}^{\left[n_{\alpha}\right]}\right\}$. Hence, $\left\{\mathbf{P}_{k} \leq \alpha I\right\}=\biguplus_{i=0}^{n_{\alpha}} F_{k}^{[i]}$. It follows from Proposition 1 that $\mathbb{P}_{k}\left(\left\{\mathbf{P}_{k} \leq \alpha I\right\}\right)=\mathbb{P}_{k}\left(\biguplus_{i=0}^{n_{\alpha}} F_{k}^{[i]}\right)=\sum_{i=0}^{n_{\alpha}} \mathbb{P}_{k}\left(F_{k}^{[i]}\right)=$ $\sum_{i=0}^{n_{\alpha}} \gamma \bar{\gamma}^{i}=1-\bar{\gamma}^{n_{\alpha}+1}$

Proof of Lemma 8: Taking mathematical expectation of (8) yields

$$
\mathbb{E}\left[\mathbf{P}_{k+1}\right]=\bar{\gamma}\left(A \mathbb{E}\left[\mathbf{P}_{k}\right] A^{\prime}+Q+U_{k}\right)+\gamma S_{k+1} .
$$

From (9), (10), and (11),

$$
\begin{aligned}
P_{k+1}^{[i+1]} & =\Psi_{k+1}^{[i+1]}\left(S_{k-i}\right) \\
& =\psi_{(k+1)-1} \circ \psi_{(k+1)-2} \circ \cdots \circ \psi_{(k+1)-(i+1)}\left(S_{k-i}\right) \\
& =\psi_{k}\left(\Psi_{k}^{[i]}\left(S_{k-i}\right)\right) \\
& =\psi_{k}\left(P_{k}^{[i]}\right)=A P_{k}^{[i]} A^{\prime}+Q+U_{k} .
\end{aligned}
$$

In the following, we prove Lemma 8 by mathematical induction. For $k=1$, from (8), $\mathbf{P}_{1}=\gamma_{1} S_{1}+\left(1-\gamma_{1}\right)\left(A P_{0} A^{\prime}+\right.$ $\left.Q+U_{0}\right) . \mathbf{P}_{1}$ takes the value $S_{1}=\Psi_{1}^{[0]}\left(S_{1}\right)=P_{1}^{[0]}$ at $\gamma_{1}=1$, and $A P_{0} A^{\prime}+Q+U_{0}=\psi_{0}\left(S_{0}\right)=\Psi_{1}^{[1]}\left(S_{0}\right)=P_{1}^{[1]}$ at $\gamma_{1}=0$. Thus, $\mathbb{E}\left[\mathbf{P}_{1}\right]=P_{1}^{[0]} \mathbb{P}_{\gamma}\left(\left\{\gamma_{1}=1\right\}\right)+P_{1}^{[1]} \mathbb{P}_{\gamma}\left(\left\{\gamma_{1}=0\right\}\right)=$ $P_{1}^{[0]} \gamma+P_{1}^{[1]} \bar{\gamma}$. Clearly, Lemma 8 holds at $k=1$.

Suppose that Lemma 8 is true for $1, \ldots, k$. From (38),

$$
\begin{aligned}
\mathbb{E}\left[\mathbf{P}_{k+1}\right]= & \bar{\gamma}\left(A \mathbb{E}\left[\mathbf{P}_{k}\right] A^{\prime}+Q+U_{k}\right)+\gamma S_{k+1} \\
\stackrel{(a)}{=} & \bar{\gamma}\left(A\left(\sum_{n=0}^{k-1} P_{k}^{[n]} \gamma \bar{\gamma}^{n}+P_{k}^{[k]} \bar{\gamma}^{k}\right) A^{\prime}\right. \\
& \left.+\left(\sum_{n=0}^{k-1} \gamma \bar{\gamma}^{n}+\bar{\gamma}^{k}\right)\left(Q+U_{k}\right)\right)+\gamma S_{k+1} \\
\stackrel{(b)}{=} & \sum_{n=0}^{k-1} \gamma \bar{\gamma}^{n+1} P_{k+1}^{[n+1]}+P_{k+1}^{[k+1]} \bar{\gamma}^{k+1}+\gamma P_{k+1}^{[0]} \\
= & \sum_{n=0}^{k} \gamma \bar{\gamma}^{n} P_{k+1}^{[n]}+P_{k+1}^{[k+1]} \bar{\gamma}^{k+1},
\end{aligned}
$$

where $(a)$ is obtained by using the hypothesis and noting that $\sum_{n=0}^{k-1} \gamma \bar{\gamma}^{n}+\bar{\gamma}^{k}=1$, and (b) is obtained by using (39) and noting that $P_{k+1}^{[0]}=\Psi_{k+1}^{[0]}\left(S_{k+1}\right)=S_{k+1}$.

Hence, (38) is true for $k+1$, which completes the proof.

Proof of Lemma 9: This lemma is proved by mathematical induction. Clearly, (24) is true for $k=N$. Suppose that (24) is true for $k+1$. Then we check $V_{k}$ as follows.

$$
\begin{aligned}
& V_{k}\left(x_{k}\right) \\
= & \min _{u_{k}} \mathbb{E}\left[x_{k}^{\prime} W_{k} x_{k}+\nu_{k} u_{k}^{\prime} \Lambda_{k} u_{k}+V_{k+1}\left(x_{k+1}\right) \mid \mathcal{I}_{k}\right] \\
\stackrel{(a)}{=} & \min _{u_{k}} \mathbb{E}\left[x_{k}^{\prime}\left(W_{k}+A^{\prime} M_{k+1} A\right) x_{k} \mid \mathcal{I}_{k}\right] \\
& +\bar{\gamma} \operatorname{tr}\left(H_{k+1}\left(A \mathbf{P}_{k} A^{\prime}+Q+\bar{\nu} \nu B u_{k} u_{k}^{\prime} B^{\prime}\right)\right) \\
& +\nu u_{k}^{\prime}\left(\Lambda_{k}+B^{\prime} M_{k+1} B\right) u_{k}+2 \nu u_{k}^{\prime} B^{\prime} M_{k+1} A \hat{\mathbf{x}}_{k} \\
& +\operatorname{tr}\left(\Delta_{k+1}+M_{k+1} Q+\gamma H_{k+1} S_{k+1}\right) \\
= & \min _{u_{k}} \mathbb{E}\left[x_{k}^{\prime}\left(W_{k}+A^{\prime} M_{k+1} A\right) x_{k} \mid \mathcal{I}_{k}\right]+2 \nu u_{k}^{\prime} B^{\prime} M_{k+1} A \hat{\mathbf{x}}_{k} \\
& +\nu u_{k}^{\prime}\left(\Lambda_{k}+B^{\prime}\left(M_{k+1}+\bar{\gamma} \bar{\nu} H_{k+1}\right) B\right) u_{k}+\operatorname{tr}\left(\Delta_{k+1}\right. \\
& \left.+M_{k+1} Q+\bar{\gamma} H_{k+1}\left(A \mathbf{P}_{k} A^{\prime}+Q\right)+\gamma H_{k+1} S_{k+1}\right)(40)
\end{aligned}
$$

where $(a)$ is obtained by using (21), (24), and some algebraic manipulations. Differentiating $V_{k}\left(x_{k}\right)$ with respect to $u_{k}$ and 
solving $\frac{\partial V_{k}}{\partial u_{k}}=0$ yield the minimizer $u_{k}^{*}=L_{k} \hat{\mathbf{x}}_{k}$ where $L_{k}=$ $-\left(\Lambda_{k}+B^{\prime}\left(\alpha M_{k+1}+\bar{\alpha} T_{k+1}\right) B\right)^{-1} B^{\prime} M_{k+1} A$. By substituting $u_{k}^{*}$ back to (40) and then using (34), we have

$$
\begin{aligned}
& V_{k}\left(x_{k}\right) \stackrel{(a)}{=} \mathbb{E}\left[x_{k}^{\prime} M_{k} x_{k} \mid \mathcal{I}_{k}\right] \\
&+\operatorname{tr}\left(\left(A^{\prime} M_{k+1} A+W_{k}-M_{k}\right) \mathbf{P}_{k}\right) \\
&+\bar{\gamma} \operatorname{tr}\left(A^{\prime} H_{k+1} A \mathbf{P}_{k}\right)+\operatorname{tr}\left(\gamma H_{k+1} S_{k+1}\right) \\
&+\operatorname{tr}\left(\Delta_{k+1}\right)+\operatorname{tr}\left(M_{k+1} Q\right)+\bar{\gamma} \operatorname{tr}\left(H_{k+1} Q\right) \\
& \stackrel{(b)}{=} \mathbb{E}\left[x_{k}^{\prime} M_{k} x_{k} \mid \mathcal{I}_{k}\right]+\operatorname{tr}\left(H_{k} \mathbf{P}_{k}\right)+\operatorname{tr}\left(\Delta_{k}\right)
\end{aligned}
$$

where $(a)$ is obtained by $(25 a)(25 b)$, and $(b)$ is obtained by (25c). Thus, (24) holds for $V_{k}$. The proof is completed.

\section{REFERENCES}

[1] J. P. Hespanha, P. Naghshtabrizi, and Y. Xu, "A survey of recent results in networked control systems," Proceedings of the IEEE, vol. 95, no. 1, p. 138, 2007.

[2] J. Zhang, Y. Lin, and P. Shi, "Output tracking control of networked control systems via delay compensation controllers," Automatica, vol. 57, pp. 85-92, 2015.

[3] P. Shi, H. Wang, and C.-C. Lim, "Network-based event-triggered control for singular systems with quantizations," Industrial Electronics, IEEE Transactions on, doi:10.1109/TIE.2015.2475515.

[4] P. Shi, X. Luan, and F. Liu, "Filtering for discrete-time systems with stochastic incomplete measurement and mixed delays," Industrial Electronics, IEEE Transactions on, vol. 59, no. 6, pp. 2732-2739, 2012.

[5] H. Yan, F. Qian, F. Yang, and H. Shi, " $h_{\infty}$ filtering for nonlinear networked systems with randomly occurring distributed delays, missing measurements and sensor saturation," Information Sciences, vol. 370371, pp. 772-782, 2016.

[6] I. F. Akyildiz, T. Melodia, and K. R. Chowdury, "Wireless multimedia sensor networks: A survey," Wireless Communications, IEEE, vol. 14, no. 6, pp. 32-39, 2007.

[7] B. Sinopoli, L. Schenato, M. Franceschetti, K. Poolla, and S. Sastry, "Optimal linear LQG control over lossy networks without packet acknowledgment," Asian Journal of Control, vol. 10, no. 1, pp. 3-13, 2008.

[8] E. Garone, B. Sinopoli, and A. Casavola, "LQG control over lossy TCPlike networks with probabilistic packet acknowledgements," International Journal of Systems, Control and Communications, vol. 2, no. 1, pp. 55-81, 2010.

[9] M. Kögel, R. Blind, F. Allgöwer, and R. Findeisen, "Optimal and optimal-linear control over lossy, distributed networks," in Proc. 18th IFAC World Congress, 2011, pp. 13239-13244.

[10] N. J. Ploplys, P. A. Kawka, and A. G. Alleyne, "Closed-loop control over wireless networks," Control Systems, IEEE, vol. 24, no. 3, pp. 58-71, 2004.

[11] H. Lin, H. Su, P. Shi, R. Lu, and Z.-G. Wu, "Estimation and LQG control over unreliable network with acknowledgment randomly lost," IEEE Trans. Cybern., 2016, DOI:10.1109/TCYB.2016.2597259.

[12] H. Lin, H. Su, Z. Shu, Z.-G. Wu, and Y. Xu, "Optimal estimation for networked control systems with intermittent inputs without acknowledgement," in Proc. 19th IFAC World Congress, 2014, pp. 5017-5022.

[13] - "Optimal estimation in UDP-like networked control systems with intermittent inputs: Stability analysis and suboptimal filter design," IEEE Trans. Autom. Control, vol. 61, no. 7, pp. 1794-1809, 2016.

[14] L. Schenato, B. Sinopoli, M. Franceschetti, K. Poolla, and S. S. Sastry, "Foundations of control and estimation over lossy networks," Proc. the IEEE, vol. 95, no. 1, pp. 163-187, 2007.

[15] H. Lin, H. Su, P. Shi, R. Lu, and Z.-G. Wu, "LQG control for networked control systems over packet drop links without packet acknowledgment," Journal of the Franklin Institute, vol. 352, no. 11, pp. 5042-5060, 2015.

[16] M. Moayedi, Y. K. Foo, and Y. C. Soh, "Networked LQG control over unreliable channels," International Journal of Robust and Nonlinear Control, vol. 23, no. 2, pp. 167-189, 2013.

[17] S. Veleva, D. Davcev, and M. Kacarska, "Wireless smart platform for home energy management system," in Innovative Smart Grid Technologies (ISGT Europe), 2011 2nd IEEE PES International Conference and Exhibition on. IEEE, 2011, pp. 1-8.

[18] R. Frank, Understanding smart sensors. IOP Publishing, 2000.

[19] N. Bressan, L. Bazzaco, N. Bui, P. Casari, L. Vangelista, and M. Zorzi, "The deployment of a smart monitoring system using wireless sensor and actuator networks," in Smart Grid Communications (SmartGridComm), 2010 First IEEE International Conference on. IEEE, 2010, pp. 49-54.

[20] L. Shi, P. Cheng, and J. Chen, "Sensor data scheduling for optimal state estimation with communication energy constraint," Automatica, vol. 47, no. 8, pp. 1693-1698, 2011.

[21] D. Shi, T. Chen, and L. Shi, "Event-triggered maximum likelihood state estimation," Automatica, vol. 50, no. 1, pp. 247-254, 2014.

[22] T. Sui, K. You, M. Fu, and D. Marelli, "Stability of MMSE state estimators over lossy networks using linear coding," Automatica, vol. 51, pp. 167-174, 2015.

[23] H. Lin, Z. Xu, H. Su, Y. Xu, and Z. Wu, "Fast filtering algorithm for state estimation of lossy networks," IET Control Theory \& Applications, vol. 8, no. 18, pp. 2316-2324, 2014.

[24] J. Baillieul and P. J. Antsaklis, "Control and communication challenges in networked real-time systems," Proceedings of the IEEE, vol. 95, no. 1, pp. 9-28, 2007.

[25] B. Sinopoli, L. Schenato, M. Franceschetti, K. Poolla, M. I. Jordan, and S. S. Sastry, "Kalman filtering with intermittent observations," IEEE Trans. Automat. Control, vol. 49, no. 9, pp. 1453-1464, 2004.

[26] K. You, M. Fu, and L. Xie, "Mean square stability for Kalman filtering with Markovian packet losses," Automatica, vol. 47, no. 12, pp. 26472657, 2011.

[27] S. Kar, B. Sinopoli, and J. M. Moura, "Kalman filtering with intermittent observations: Weak convergence to a stationary distribution," Automatic Control, IEEE Transactions on, vol. 57, no. 2, pp. 405-420, 2012.

[28] A. Censi, "Kalman filtering with intermittent observations: convergence for semi-markov chains and an intrinsic performance measure," Automatic Control, IEEE Transactions on, vol. 56, no. 2, pp. 376-381, 2011.

[29] M. Epstein, L. Shi, A. Tiwari, and R. M. Murray, "Probabilistic performance of state estimation across a lossy network," Automatica, vol. 44, no. 12, pp. 3046-3053, 2008.

[30] L. Shi, M. Epstein, and R. M. Murray, "Kalman filtering over a packetdropping network: a probabilistic perspective," Automatic Control, IEEE Transactions on, vol. 55, no. 3, pp. 594-604, 2010.

[31] O. C. Imer, S. Yüksel, and T. Başar, "Optimal control of LTI systems over unreliable communication links," Automatica, vol. 42, no. 9, pp. $1429-1439,2006$

[32] K. Plarre and F. Bullo, "On Kalman filtering for detectable systems with intermittent observations," IEEE Trans. Automat. Control, vol. 54, no. 2, pp. 386-390, 2009.

[33] M. Huang and S. Dey, "Stability of Kalman filtering with Markovian packet losses," Automatica, vol. 43, no. 4, pp. 598-607, 2007.

[34] E. R. Rohr, D. Marelli, and M. Fu, "Kalman filtering with intermittent observations: On the boundedness of the expected error covariance," Automatic Control, IEEE Transactions on, vol. 59, no. 10, pp. 2724$2738,2014$.

[35] Y. Mo and B. Sinopoli, "Kalman filtering with intermittent observations: tail distribution and critical value," IEEE Trans. Automat. Control, vol. 57, no. 3, pp. 677-689, 2012.

[36] E. Garone, B. Sinopoli, A. Goldsmith, and A. Casavola, "LQG control for MIMO systems over multiple erasure channels with perfect acknowledgment," IEEE Trans. Automat. Control, vol. 57, no. 2, pp. 450-456, 2012.

[37] H. Xu, S. Jagannathan, and F. L. Lewis, "Stochastic optimal control of unknown linear networked control system in the presence of random delays and packet losses," Automatica, vol. 48, no. 6, pp. 1017-1030, 2012.

[38] L. Schenato, "Optimal estimation in networked control systems subject to random delay and packet drop," Automatic Control, IEEE Transactions on, vol. 53, no. 5, pp. 1311-1317, 2008.

[39] V. Gupta, B. Hassibi, and R. M. Murray, "Optimal LQG control across packet-dropping links," Systems \& Control Letters, vol. 56, no. 6, pp. 439-446, 2007.

[40] P. Billingsley, Probability and Measure. John Wiley \& Sons, 2008.

[41] R. B. Ash and C. Doleans-Dade, Probability and Measure Theory. Academic Press, 2000.

[42] P. Lancaster and L. Rodman, Algebraic Riccati Equations. Oxford University Press, 1995.

[43] Q. Qian, D. Dongmei, L. Feng, and T. Yongchuan, "Stabilization of the double inverted pendulum based on discrete-time model predictive control," in Automation and Logistics (ICAL), 2011 IEEE International Conference on. IEEE, 2011, pp. 243-247.

[44] H. Zhang, Q. Hong, H. Yan, F. Yang, and G. Guo, "Event-based distributed $H_{\infty}$ filtering networks of 2DOF quarter-car suspension systems," IEEE Trans. Ind. Informat., 2016, DOI: 10.1109/TII.2016.2569566.

[45] X. Wang and M. D. Lemmon, "Event-triggering in distributed networked control systems," IEEE Trans. Automat. Control, vol. 56, no. 3, pp. 586- 
601, 2011.

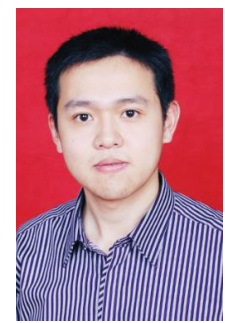

Hong Lin was born in 1980. He received the B.S. and M.S. degrees from Fuzhou University, Fuzhou, China, in 2003 and 2006, respectively, and the Ph.D. degree from Zhejiang University, Hangzhou, China, in 2016. He was a lecturer with the Department of Information Technology, Concord College Fujian Normal University, Fuzhou, China, from 2007 to 2012. He is currently a Postdoctoral Researcher at the Department of Mechanical Engineering, The University of Hong Kong, Hong Kong, China. His current research interests include networked control systems, estimator design, and optimal control.

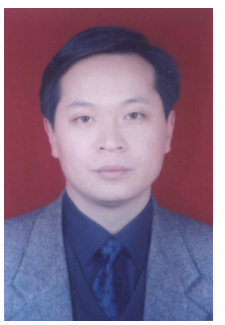

Hongye Su (SM'14) was born in 1969. He received the B.S. degree in industrial automation from the Nanjing University of Chemical Technology, Nanjing, China, in 1990, and the M.S. and Ph.D. degrees from Zhejiang University, Hangzhou, China, in 1993 and 1995, respectively.

He was a lecturer with the Department of Chemical Engineering, Zhejiang University, Hangzhou, China, from 1995 to 1997. From 1998 to 2000, he was an Associate Professor with the Institute of Advanced Process Control, Zhejiang University, Hangzhou, China. Currently, he is a Professor with the Institute of CyberSystems and Control, Zhejiang University, Hangzhou, China. His current research interests include the robust control, time-delay systems, and advanced process control theory and applications.

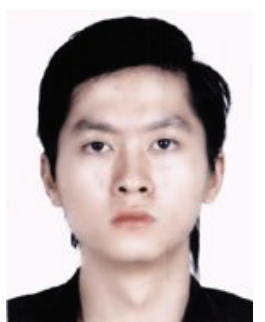

Zhan Shu was born in Nanchang, Jiangxi province, China, in 1982. He received his B.Eng. degree in Automation from Huazhong University of Science and Technology (HUST) in 2003, and the Ph.D. degree in Control Engineering from The University of Hong Kong (HKU) in 2008. From 2008 to 2009, he worked in HKU as a research assistant/associate. From 2009 to 2011, he was a postdoctoral researcher in the Hamilton Institute, National University of Ireland, Maynooth. He joined the University of Southampton, U.K., in 2011, taking a lectureship in the Faculty of Engineering and the Environment. He is a Member of IEEE and an invited reviewer of Mathematical Review of the American Mathematical Society. He serves as an editorial member for The Open Automation and Control Systems Journal, an Associate Editor for Mathematical Problems in Engineering, an Associate Editor for Asian Journal of Control, an Associate Editor for Journal of The Franklin Institute, and a member of the IEEE Control Systems Society Conference Editorial Board. His current research interests include hybrid systems, stochastic systems, positive systems, delay systems, robust control, decentralized control, estimation and filtering, model free control, control application in energy systems, systems biology with emphasis on immunology.

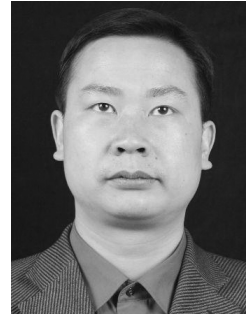

Renquan Lu (M'08) received the Ph.D. degree in control science and engineering from Zhejiang University, Hangzhou, China, in 2004.

$\mathrm{He}$ is currently a full Professor with the Institute of Information and Control, Hangzhou Dianzi University, Hangzhou. He has published more than 30 journal papers in the fields of robust control and complex systems. His research interests include robust control, singular systems, and complex systems.

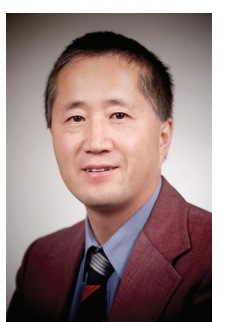

Peng Shi (M'95/SM'98/F'15) received the PhD degree in Electrical Engineering from the University of Newcastle, Australia in 1994; the PhD degree in Mathematics from the University of South Australia in 1998. He was awarded the Doctor of Science degree from the University of Glamorgan, UK in 2006, and the Doctor of Engineering degree from the University of Adelaide in 2015.

Dr Shi is a professor at the University of Adelaide, and Victoria University, Australia, and an adjunct professor at Harbin Engineering University. He was a professor at the University of Glamorgan, UK; and a senior scientist in the Defence Science and Technology Organisation, Australia. His research interests include system and control theory, computational intelligence, and operational research. He has actively served in the editorial board of a number of journals, including Automatica, IEEE Transactions on Automatic Control; IEEE Transactions on Fuzzy Systems; IEEE Transactions on Cybernetics; IEEE Transactions on Circuits and Systems-I: Regular Papers; and IEEE Access. He is a Fellow of the Institution of Engineering and Technology, and the Institute of Mathematics and its Applications. He was the Chair of Control Aerospace and Electronic Systems Chapter, IEEE South Australia Section. Currently he serves as an IEEE Distinguished Lecturer, and a Member of Australian Research Council, College of Expert.

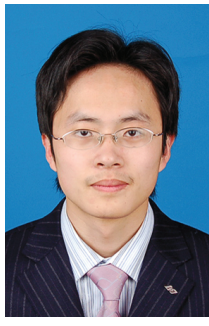

Zheng-Guang Wu was born in 1982. He received the B.S. and M.S. degrees from Zhejiang Normal University, Jinhua, China, in 2004 and 2007, respectively, and the Ph.D. degree from Zhejiang University, Hangzhou, China, in 2011.

He was a Research Associate with the Department of Mathematics, City University of Hong Kong, Hong Kong, from February 2010 to June 2010, and a Senior Research Associate with the Department of Mechanical and Biomedical Engineering, City University of Hong Kong, Hong Kong, from July 2014 to October 2014. He was a Research Associate with the Department of Mechanical Engineering, the University of Hong Kong, Hong Kong, from December 2010 to February 2011, and from March 2015 to June 2015, respectively. He was a Post-doctoral Research Associate with the Department of Electrical Engineering, Yeungnam University, Kyongsan, Republic of Korea, from September 2011 to August 2013. He is currently with the Institute of Cyber-Systems and Control, Zhejiang University, Hangzhou, China. He currently serves as an Associate Editor for IEEE Access and an Editorial Board Member for Neurocomputing. He is a very active reviewer for many international journals. His current research interests include Markov jump systems, sampled-data control systems, networked control systems, and timedelay systems. 